\section{Pacific Northwest}

National Laboratory

Operated by Battelle for the

U.S. Department of Energy

\title{
Adaptation of Machine Fluid Analysis for Manufacturing Final Report
}

AF Pardini

August 2005

Prepared for the U.S. Department of Energy

under Contract DE-AC05-76RL01830 


\title{
DISCLAIMER
}

This report was prepared as an account of work sponsored by an agency of the United States Government. Neither the United States Government nor any agency thereof, nor Battelle Memorial Institute, nor any of their employees, makes any warranty, express or implied, or assumes any legal liability or responsibility for the accuracy, completeness, or usefulness of any information, apparatus, product, or process disclosed, or represents that its use would not infringe privately owned rights. Reference herein to any specific commercial product, process, or service by trade name, trademark, manufacturer, or otherwise does not necessarily constitute or imply its endorsement, recommendation, or favoring by the United States Government or any agency thereof, or Battelle Memorial Institute. The views and opinions of authors expressed herein do not necessarily state or reflect those of the United States Government or any agency thereof.

\author{
PACIFIC NORTHWEST NATIONAL LABORATORY \\ operated by \\ BATTELLE \\ for the \\ UNITED STATES DEPARTMENT OF ENERGY \\ under Contract DE-AC05-76RL01830
}

Printed in the United States of America
Available to DOE and DOE contractors from the Office of Scientific and Technical Information,
P.O. Box 62, Oak Ridge, TN 37831-0062;
ph: (865) 576-8401
fax: $(865)$ 576-5728
email: reports@adonis.osti.gov

\begin{abstract}
Available to the public from the National Technical Information Service, U.S. Department of Commerce, 5285 Port Royal Rd., Springfield, VA 22161 ph: (800) 553-6847 fax: $(703) 605-6900$ email: orders@ntis.fedworld.gov online ordering: http://www.ntis.gov/ordering.htm
\end{abstract}

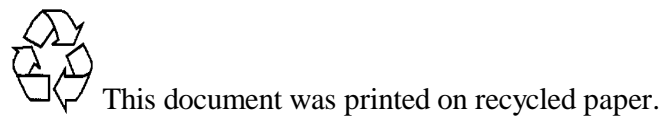




\section{Adaptation of Machine Fluid Analysis for Manufacturing Final Report Project 44213/46125}

AF Pardini

August 2005

Prepared for

the U.S. Department of Energy

under Contract DE-AC05-76RL01830

Pacific Northwest National Laboratory

Richland, Washington 99352 


\section{Summary}

This report describes the technical work that was adapted from recent DOE supported advances in automated on-site machine fluid technology for use in the manufacturing industry. This work was conducted by Pacific Northwest National Laboratory with industrial contributions by Package Corporation of America and subcontracted work by Belhaven Applied Technologies.

The development of the machine fluid analysis systems began in fiscal year 1999 at the Pacific Northwest National Laboratory (PNNL) under the DOE OIT Mining Industry of the Future program. It was anticipated that the program would last 36 months; however it was extended at no additional cost until the end of fiscal year 2004. Filtration and lubricant suppliers to the pulp and paper industry had noted the recent accomplishments by PNNL and its industrial partners in the DOE OIT Mining Industry of the Future Program, and asked for assistance in adapting this DOE-funded technology to the pulp and paper industry. A new project was started in fiscal year 2002 to support this request. This project was titled "Adaptation of Machine Fluid Analysis for Manufacturing".

Technology developed during this project was selected for use in the pulp and paper industry. Building on technology developed for the mining industry, new control and user interface software for an on-site system has been re-written to run in the National Instrument Lab View environment. The software has been tested and rigorous quality assurance checks have been applied. Other advances in technology include a miniature XRF sensor that is smaller and more sensitive than the earlier version, and a closecoupled infrared sensor which has 22 channels and can be configured to meet specific equipment requirements.

Original deliverables for the project included an on-site machine fluid analysis system tailored to requirements of a large paper mill and a final report of the system's on-site performance including a cost benefit proposal. It was anticipated that a demonstration of the system would be performed at the Packaging Corporation of America (PCA) paper mill in Counce, Tennessee pending management approval. However, the current stand-alone system is at the Pall Corporation site in New York awaiting deployment at the PCA Valdosta, GA. facility. 


\section{Contents}

PROJECT SUMMARY ..................................................................................... i

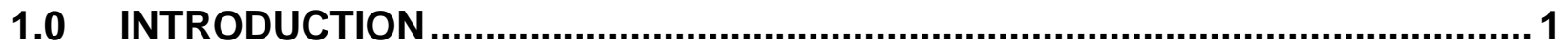

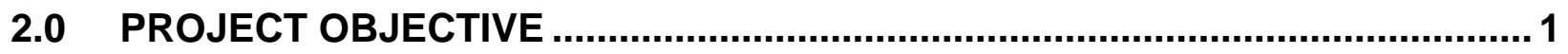

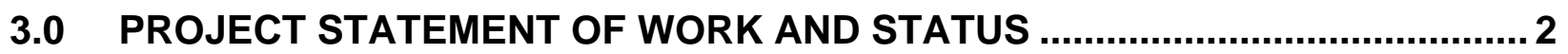

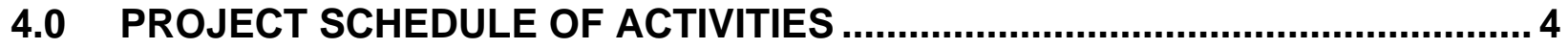

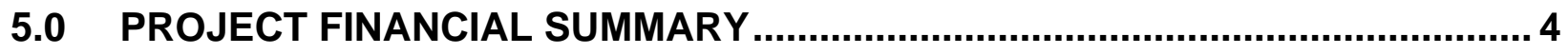

6.0 PROJECT HIGHLIGHTS AND SYSTEM COMPONENTS …............................ 5

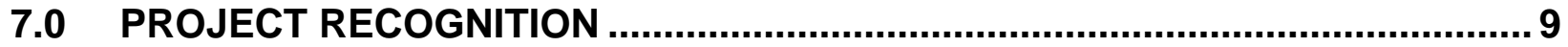

8.0 PROJECT COMMERCIALIZATION OUTLOOK............................................ 10

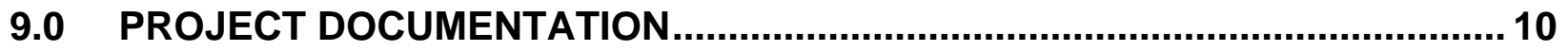




\section{Figures}

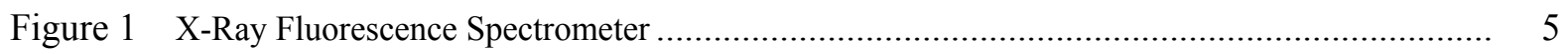

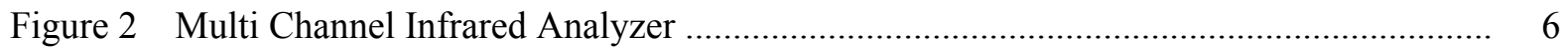

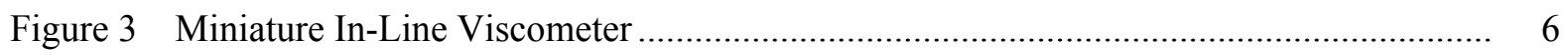

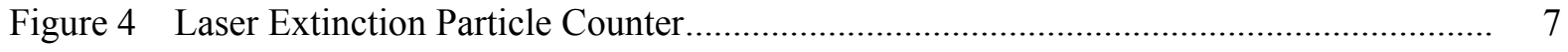

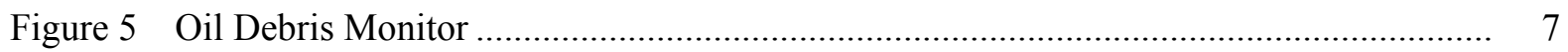

Figure 6 Sample Bottle Handling/Routing/and Flow Control Module ........................................ 8 


\subsection{Introduction}

The development of the machine fluid analysis systems began in fiscal year 1999 at the Pacific Northwest National Laboratory (PNNL) under the DOE OIT Mining Industry of the Future program. It was anticipated that the program would last 36 months; however it was extended at no additional cost until the end of fiscal year 2004. Filtration and lubricant suppliers to the pulp and paper industry had noted the recent accomplishments by PNNL and its industrial partners in the DOE OIT Mining Industry of the Future Program, and asked for assistance in adapting this DOE-funded technology to the pulp and paper industry. A new project was started in fiscal year 2002 to support this request. This project was titled "Adaptation of Machine Fluid Analysis for Manufacturing".

Paper manufacture involves the operation of extensive and complex high-speed process lines that utilize hydraulic systems and rely heavily on proper lubrication. Failure of bearings anywhere in the line can lead quickly to failure of major components resulting in shut-down of the entire line at great expense to the owners and operators. Successful adaptation of this DOE-funded technology could result in substantial reductions in operating costs as well as reduced use of lubricants and extended equipment life. Owners and operators of high-end manufacturing equipment and process equipment will be among the early adopters of automated in-line multi-parameter analytical equipment for monitoring of lubrication and power fluids.

\subsection{Project Objective}

The objective of this project was to adapt automated on-site lubrications power fluid analysis capability developed under the DOE OIT Mining Industry of the Future program for use in the pulp and paper industry. Under this proposal PNNL, Belhaven Applied Technologies, Oil Analysis Laboratory, and Pall Corporation would design, fabricate, install and demonstrate a tailored automated on-site oil analysis system. The system would provide near real-time diagnostic/prognostic information on equipment health.

Among the needed adaptations to the DOE-funded technology for use on pulp and paper manufacturing process lines include development of automated particle counting that can be related to the new ISO 111701 particle fluid cleanliness standards, development of on-site waste oil as fuel analysis protocols, development of tailored software for control data acquisition equipment health status monitoring and revised on-condition maintenance protocols. Development of ergonomically sound user software and sample handling methods will also be required, as will integration of the data into the maintenance practices and procedures of the plant. It was anticipated that a demonstration of the system would be performed at the Packaging Corporation of America (PCA) paper mill in Counce, Tennessee pending management approval. However, the current stand-alone system is at the Pall Corporation site in New York awaiting deployment at the PCA Valdosta, GA. facility. 


\subsection{Project Statement of Work and Status}

Each of the tasks below describes the work that was to be accomplished and the status as of this report writing.

\subsection{Task 1: Pulp and paper industry needs assessment}

Specific machine fluid requirements relevant to the pulp and paper industry will be determined. This task will include review and listing of all power fluids, lubrication products and fuels utilized in the PCA operation. Review of present machine fluid analysis operations and alert alarm protocols will be carried out.

Current Status: This work has been completed. A needs assessment was performed during fiscal year 2002 which provided the necessary requirements relative to the pulp and paper industry to allow PNNL to development the system parameters.

\subsection{Task 2: On-site system specification and cost benefit analysis}

3.2.1 Review present machine fluid analysis schedules and determine the analytical modules required to support present and anticipated upgraded maintenance procedures.

Current Status: This work has been completed. Reference Attachment 1.

3.2.2 Perform sensitivity analysis to determine most effective use of onsite capability and specific capabilities required.

Current Status: This work has been completed. Reference Attachment 1.

3.2.3 Perform cost benefit analysis for on-site system by hardware module and software module.

Current Status: This work has been completed. Reference Attachment 1.

\subsection{Task 3: System design}

Based on needs assessment and cost benefit trade studies outcome, design system hardware and software to provide needed capabilities at the lowest cost. Design tasks include development of tailored user interface software, development of controller software, development of suitable data base structure.

Current Status: This work has been completed. Reference Attachment 2.

\subsection{Task 4: Procurement}

PNNL will procure hardware and software components required as determined by preliminary design specifications.

Current Status: This work has been completed. 


\subsection{Task 5: Assembly, software modification and bench testing}

3.5.1 Assemble the system as designed, install software and bench test system at Belhaven Applied Technologies.

Current Status: This work has been completed. Reference Attachment 3.

3.5.2 Modify software as required based on specifications developed under Task 3.3.

Current Status: This work has been completed. Reference Attachment 3.

3.5.2 Demonstrate suitable completion of test protocols and suitable performance based on tests of standards and machine fluid samples provided by Oil Analysis Laboratory (OAL) in Spokane, Washington.

Current Status: This work has been completed. Reference Attachment 3.

\subsection{Task 6: Installation and field-testing PCA site in Counce TN}

Upon completion of bench testing and pending management approval, PNNL and PCA staff will install the stand-alone system at the PCA plant for evaluation. Work under this task includes partial disassembly, packing shipping and re-assembly and functional testing on-site. Power and compressed air or nitrogen required for system operation will be provided by PCA.

Current Status: The stand-alone system is currently at the Pall Corporation site in New York awaiting deployment at the Valdosta, GA. facility. The completion of this work is to be determined.

\subsection{Task 7: On-Site Demonstration}

PCA and PNNL will conduct an on-site demonstration of the technology at the PCA paper mill in Counce $\mathrm{TN}$ in accordance with the schedule shown in Section 5. Work under this task includes comparison of data obtained with the on-site equipment to that obtained from commercial laboratories on aliquots of the same samples, and documentation of the direct and indirect cost and energy savings accrued from use of the technology.

Current Status: The completion of this work is to be determined.

\subsection{Task 8: Evaluation and Final Report}

PNNL and PCA will review analytical data developed by the onsite system and evaluate the benefit to operations at the Counce plant. A final report describing the technology and the cost benefit performance will be provided to DOE EE. If such benefits warrant, PCA plans to adopt this automated on-site analysis technology in other paper mill facilities.

Current Status: The completion of this work is to be determined. However, this document serves as the final report that will be submitted to DOE EE. 


\subsection{Project Schedule of Activities}

\section{Planned Milestones}

- Onsite System Specification

May 2002

- Perform System Design

June 2002

- Assembly, Software Modification and Bench Testing

August 2002

- On-site Demonstration

September 2002

\subsection{Project Financial Summary}

Total for projects 44213 Adaptation of Machine Fluid Analysis for Manufacturing and 46125 Adaptation of Machine Fluid Analysis for Manufacturing II is $\$ 198 \mathrm{~K}$.

Project 44213 costs were: $\$ 139 \mathrm{~K}$

Project 46125 costs were: $\$ 59 \mathrm{~K}$ 


\subsection{Project Highlights and System Components}

Technology developed during this project was selected for use in the pulp and paper industry. Building on technology developed for the mining industry, new control and user interface software for an on-site system has been re-written to run in the National Instrument Lab View environment. The software has been tested and rigorous quality assurance checks have been applied. Other advances in technology include a miniature XRF sensor that is smaller and more sensitive than the earlier version, and a close-coupled infrared sensor which has 22 channels and can be configured to meet specific equipment requirements.

Equipment that has been adapted to provide on-site characterization of fluids includes:

\section{$X R F$ Spectrometer}

Energy dispersive $\mathrm{x}$-ray spectrometer comprising a ${ }^{109} \mathrm{Cd}$ source, and $\mathrm{x}$-ray detector with 256 or 512 channel multichannel analyzer for quantitative determination of wear or additive metals including $\mathrm{Fe}, \mathrm{Cr}, \mathrm{Ni}, \mathrm{Mo}, \mathrm{Cu}, \mathrm{Pb}, \mathrm{Zn}$, Ag, Sn, V, Co, and Ti. (EDAX CT 5000).

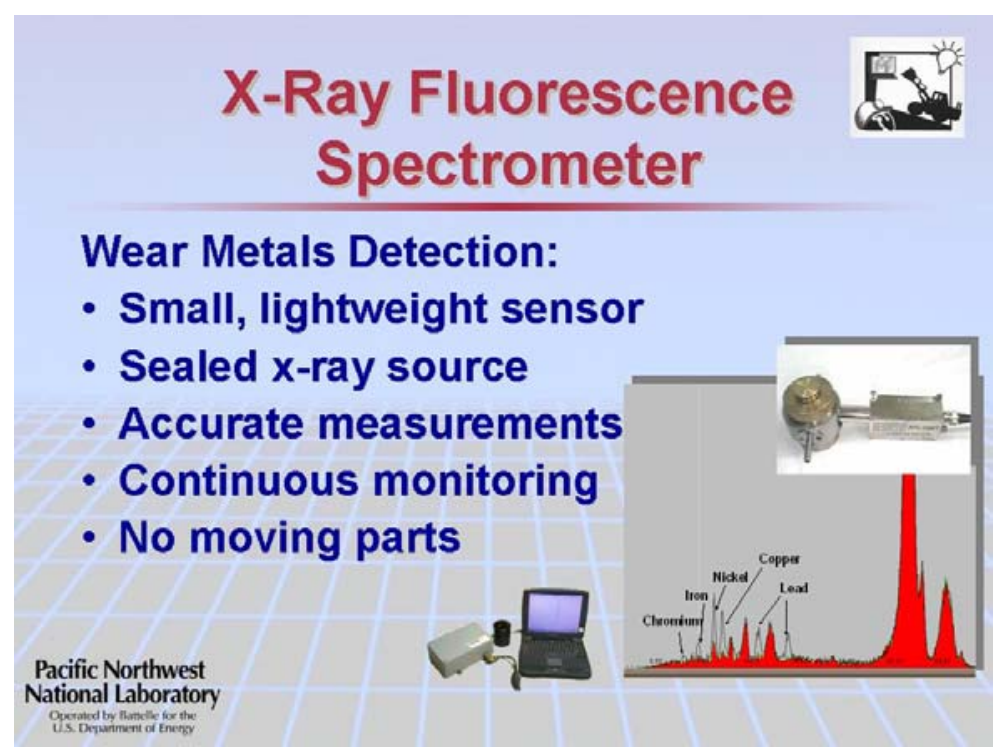

Figure 1 X-Ray Fluorescence Spectrometer 


\section{NDIR Infrared Analyzer}

Non-dispersive multi-channel infrared system for determination of water, oxidation, ester breakdown, nitration, sulfation, and fuel dilution in crankcase and gear lube oils as well as hydraulic fluids. (FIRM 57 upgraded to ARAS48).

\section{Electromechanical Viscometer}

Miniature viscometer for determination of temperature compensated dynamic viscosity to better than $1.4 \%$ at $0.8 \%$ precision. (CASI STL 570 upgraded to ViscoPro 1500).

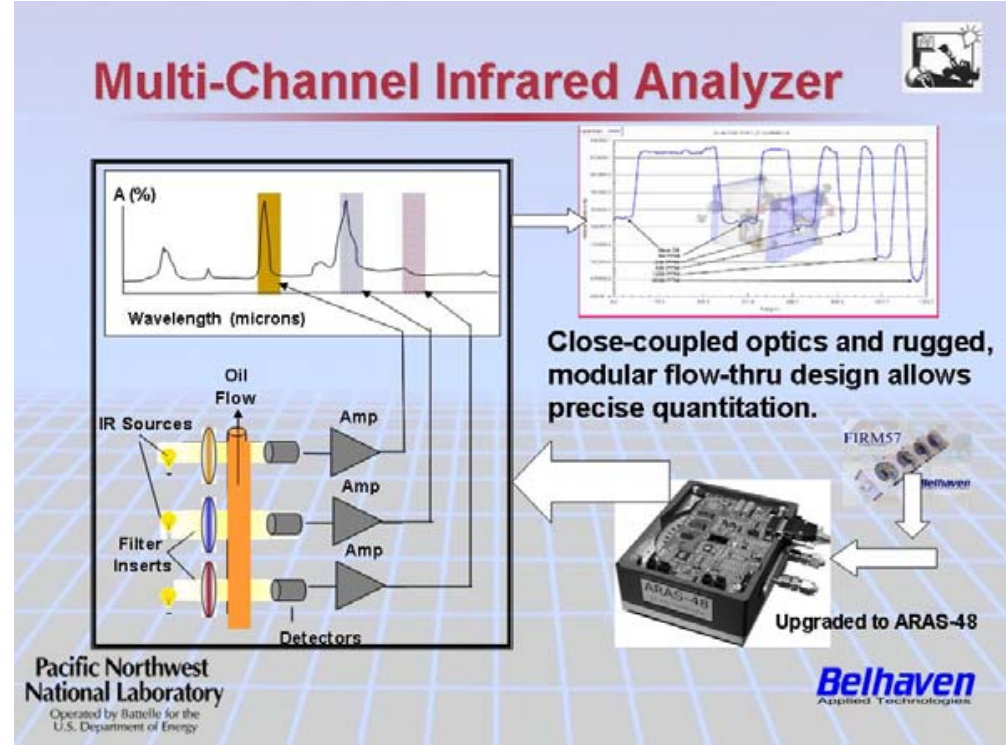

Figure 2 Multi-Channel Infrared Analyzer

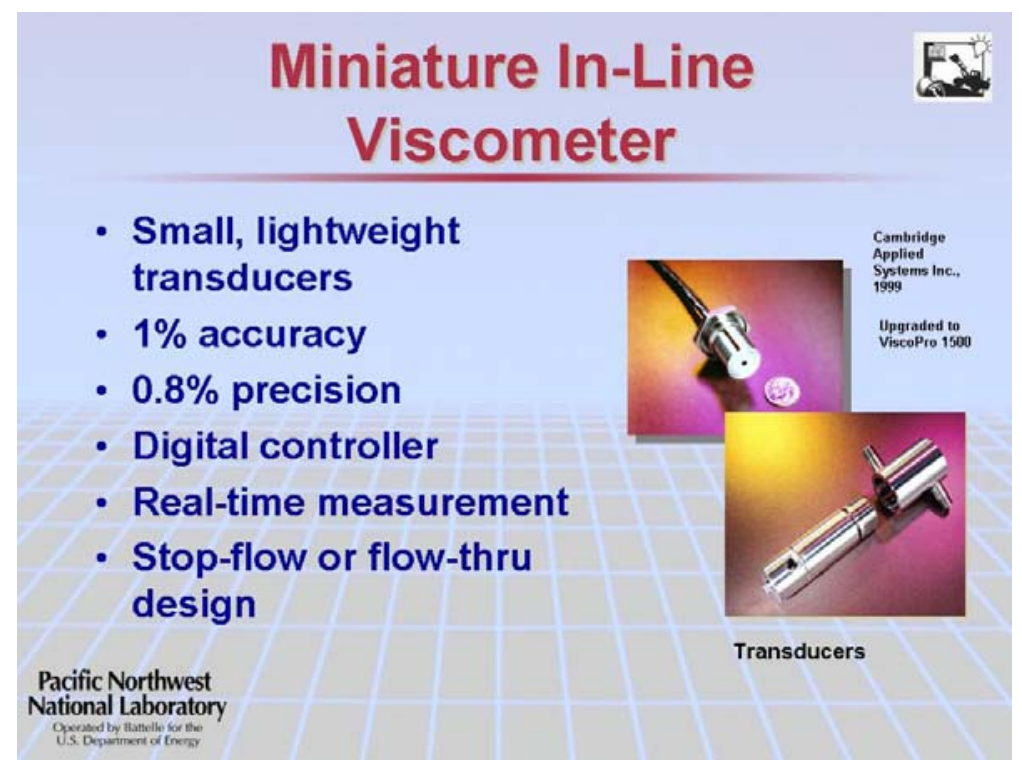

Figure 3 Miniature In-Line Viscometer 


\section{Laser Extinction Particle Counter} Determination of particles counts in eight size channels adjustable from 5 microns to $100+$ microns. (ARTI PODS Derivative).
Debris monitor. Monitors Ferrous and Non-ferrous wear particles. Minimum Ferrous particle size is 70 microns, minimum Non-ferrous particle size is 280 microns.

\section{Laser Extinction Particle Counter}

- Counts particles in 8 size ranges

from 4.9 to $100+$ microns

-Fast count rates

-High precision

-Rugged design

- Low cost solution

Pacific Northwest

National Laboratory

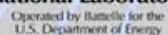

Figure 4 Laser Extinction Particle Counter

\section{Oil Debris Monitor}

- In-line system

- Determines approximate mass of ferromagnetic and non-ferromagnetic particles

- Trends particle counts and total particle mass

Pacific Northwest National Laboratory

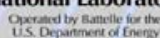

Figure 5 Oil Debris Monitor

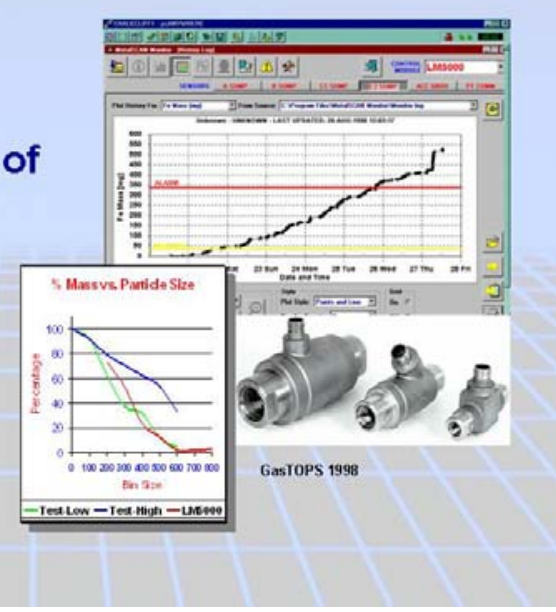

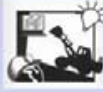

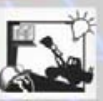


Multi-Sample Introduction System

Battelle / Belhaven / OAL System for on-site introduction of oil samples for analysis.

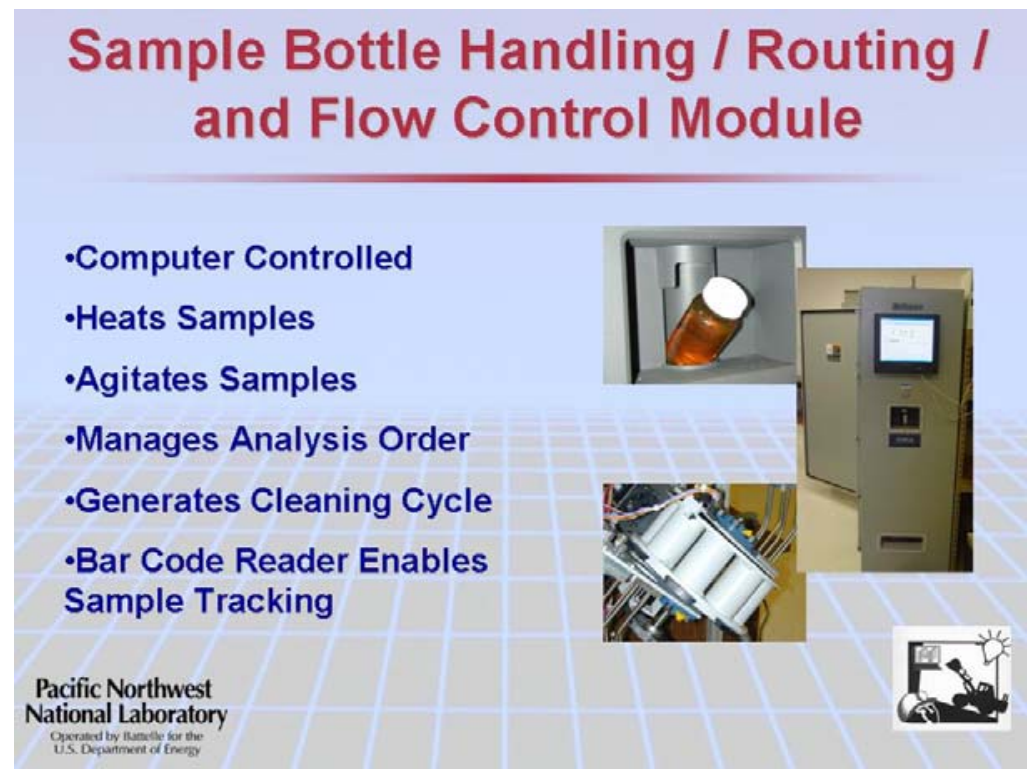

Figure 6 Sample Bottle Handling/Routing/and Flow Control Module

\section{System Command and Control Module}

Advantix 586 computer system with Battelle OASCCM software providing TCP/IP compatibility with the Internet and local user interface.

\section{Sample Acquisition and Management System}

BAT SAS 200 system for sample conditioning prior to analytical module analysis.

\section{Software:}

Bulk XRF (EDAX fundamental parameters software for "Lab-in-a-Box".

OASCCM (Battelle NT-based operating system for "Lab-in-a-Box".

Battelle "Lab-in-a-Box" User Interface

Data base software to be selected (Dingo is recommended).

AC Power Supply and Controller, System Case and Hardware 


\subsection{Project Recognition}

The development of the machine fluid analysis system has produced considerable recognition for the laboratory. Papers written and presented include:

Wilson, B.W. and Silvernail, G: "Automated In-Line Machine Fluid Analysis for Marine Diesel and Gas Turbine Engines," Proceedings: Technology Showcase 2002, JOAP International Condition Monitoring Conference, Mobile AL, April 7-11 2002.

Wilson, B.W., NH Hansen, G, Silvernail, B.Cross: "Modular System for Multi-Parameter In-Line Machine Fluid Analysis." Proceedings: Technology Showcase 2000, JOAP International Condition Monitoring Conference, Mobile, AL. April 3-6 2000. Joint Oil Analysis Program Technology Center Pensacola.

Wilson, BW and Price LS:On-Board X-Ray Fluorescence Analysis System. Lubrication and Fluid Power, Vol. 1 pp 36-38 (2000).

Wilson, B. W., N. H. Hansen, C. L. Shepard, T. J. Peters, and F. L. Greitzer: "Development of a Modular In-Situ Oil Analysis Prognostic System." Proceedings, SOLE '99 Symposium, Las Vegas, NV. 1999.

Intellectual Property that has been generated in the area of In-line Fluid Analysis include:

Apparatus and Method for Fluid Analysis

Battelle No. 12220-E

Patent No. 6,561,010

An Apparatus for Machine Fluid Analysis

Battelle No. 12092-B

Patents pending in Australia and Europe. Application nos. 16200/01 (Australia) and 00978775.5 (EPC)

Method and Apparatus to Predict the Remaining Service Life of an Operating System Battelle No. 12241-E

Patent Pending. Application nos. PCT/US00/23389 \& 10/362,717

Diagnostics/Prognostics Using Wireless Links

Battelle No. 12898-B

Patent No. 6,662,091 
Compact X-Ray Fluorescence Spectrometer and Method for Fluid Analysis

Battelle No. 13093-B

Patent No. 6,668,039

Dual X-Ray Fluorescence Spectrometer and Method for Fluid Analysis

Battelle No. 13610-E

Patent Pending. Application no. 10/420,460

\subsection{Project Commercialization Outlook}

- A non-exclusive license for manufacture, sale and use of the technology developed under this program has been granted to Belhaven Applied Technologies in Kennewick, WA.

- US Patent Number 6,561,010 for an Apparatus and method for fluid analysis was granted on May $13,2003$.

- ARTI, a small company in Grants Pass Oregon is negotiating for rights to manufacture a combined IR/particle counter system based on the technology developed under this program.

- DOE OIT project industrial partners have first-in-line rights to license background intellectual property.

\subsection{Project Documentation}

The attachments to this document provide the project documentation for the final report. 


\section{Attachment 1}

\section{Reference Task 2: On-site system specification and cost benefit analysis}


To accomplish the on-site analysis required in the Counce plant, we anticipate that the analytical and software modules listed in Table 1 will need to be developed and incorporated into the system.

Table 1 Analytical hardware and software modules required for on-site system to be used in the pulp and paper industry.

\begin{tabular}{|l|l|}
\hline Module & Function \\
\hline $\begin{array}{l}\text { XRF Elemental Analyzer } \\
\left(\text { Dual Source }{ }^{109} \mathrm{Cd} \text { and }{ }^{55} \mathrm{Fe}\right)\end{array}$ & $\begin{array}{l}\text { Determine presence and concentration of wear metals and } \\
\text { contaminants in machine fluids, and sulfur content in waste } \\
\text { oil boiler fuels. }\end{array}$ \\
\hline $\begin{array}{l}\text { Infrared Analyzer } \\
(22 \text { Channel NDIR) }\end{array}$ & $\begin{array}{l}\text { Determine water, total acid number (TAN), total base number } \\
\text { (TBN), additive, soot, glycol, oxidation, nitration and } \\
\text { sulfation }\end{array}$ \\
\hline $\begin{array}{l}\text { Laser Extinction Particle Counter } \\
(1 \text { to } 100+\text { microns) }\end{array}$ & $\begin{array}{l}\text { Determination of particle counts in specific size ranges in } \\
\text { accordance with ISO 11171. }\end{array}$ \\
\hline Viscometer & Determine kinematic viscosity at 40 or 100 degrees C \\
\hline System Controller & $\begin{array}{l}\text { Computer to control system and support user interface } \\
\text { software }\end{array}$ \\
\hline $\begin{array}{l}\text { Sample Bottle Handler / } \\
\text { Conditioning Auto Sampler }\end{array}$ & $\begin{array}{l}\text { New device to receive samples and to maintain in agitated } \\
\text { (particle-dispersed) and warm condition until aliquoted and } \\
\text { routed to the analytical modules required. }\end{array}$ \\
\hline Embedded Software Modules & All analytical modules will require embedded software \\
\hline User Interface Software & Provide for on-site and remote operation of the system \\
\hline Communications Software & Provides for data transfer and remote operation of the system \\
\hline System Controller Software & Controls analytical modules and autosampler \\
\hline
\end{tabular}

\section{Portable Lubricating Oil and Hydraulic Fluid Analysis System Specifications}

The AutOLab ${ }^{\mathrm{TM}}$ oil analysis system uses multiple field-hardened instruments to make laboratory quality measurements of oil or hydraulic fluid condition on a near real time basis. The modular approach taken with the AutOLab ${ }^{\mathrm{TM}}$ system allows the customer to choose the right combination of instruments for their needs. This approach also allows for the easy integration of new instruments as they become available. The instruments that have been used to date include:

1. Non-dispersive Infrared Analysis (NDIR),

2. X-ray Fluorescence Spectrography (XRF),

3. Electromechanical Viscometer,

4. Laser Extinction Particle Counter, and

5. In-line Debris Monitor. 
Some of the parameters that can be measured with these instruments include:

1. Water content (NDIR),

2. TAN/TBN (NDIR),

3. Additive condition (NDIR),

4. Oxidation/Sulfation (NDIR),

5. Contamination (NDIR, particle counter and XRF),

6. Elemental wear and contamination analysis (XRF),

7. Viscosity,

8. Solid particle contamination, and

9. Ferrous and non-ferrous debris particles.

The specifications for each component are listed below along with a comparison with the Joint Oil Analysis Program (JOAP) requirements for the Army. Where possible, comparisons of AutOLab ${ }^{\mathrm{TM}}$ instruments with standard oil analysis lab measurements are included.

Non-dispersive infrared analysis. Can measure infrared absorption for up to 32 channels in the 2.5 to 12 micron range (other ranges can be requested). Typical precision per band is given in Table 1 below.

\begin{tabular}{|l|l|l|l|l|}
\hline Wavelength (microns) & 0.8 & 2.9 & 5.7 & $6-9$ \\
\hline $\begin{array}{l}\text { Precision (average percent } \\
\text { error) }\end{array}$ & 0.4 & 0.013 & 0.23 & 0.62 \\
\hline
\end{tabular}

Table 1. Typical precision per band for non-dispersive infrared analyzer.

A comparison of NDIR results with standard laboratory measurements for water and TAN are given in Table 2 below. The NDIR is capable of measuring water content to $50 \mathrm{ppm}$ or less.

\begin{tabular}{|l|l|l|l|}
\hline System/Laboratory & NDIR Spectroscopy & Oil Analysis Lab* & Navy Lab** \\
\cline { 1 - 3 } Parameter & & & \\
\cline { 1 - 3 } Water Sample \#1 & $410 \mathrm{ppm}$ & $430 \mathrm{ppm}$ & $370 \mathrm{ppm}$ \\
Water Sample \#2 & $440 \mathrm{ppm}$ & $450 \mathrm{ppm}$ & $380 \mathrm{ppm}$ \\
\hline TAN Sample \#1 & $0.70 \mathrm{mg} \mathrm{KOH} / \mathrm{g}$ & $0.70 \mathrm{mg} \mathrm{KOH} / \mathrm{g}$ & $0.64 \mathrm{mg} \mathrm{KOH} / \mathrm{g}$ \\
\cline { 1 - 1 } TAN Sample \#2 & $0.77 \mathrm{mg} \mathrm{KOH} / \mathrm{g}$ & $0.70 \mathrm{mg} \mathrm{KOH} / \mathrm{g}$ & $0.64 \mathrm{mg} \mathrm{KOH} / \mathrm{g}$ \\
\hline * Data provided by Oil Analysis Lab, Spokane, Wa \\
* Water by Karl Fischer, ASTM D1744, TAN by ASTM D664 \\
\hline **Water by Karl Fischer, ASTM D6304, TAN by ASTM D974 \\
\hline
\end{tabular}

Table 2. Comparison of NDIR results with laboratory measurements. 
X-ray fluorescence spectroscopy. The standard source configuration for the XRF is ${ }^{109}$ Cadnium. There is no limit on the particle size for X-ray fluorescence spectroscopy measurements. Table 3 below lists the elemental detection limits at 10 percent precision for the standard configuration for the AutOLab ${ }^{\mathrm{TM}}$ compared with the calibration requirements for AOAP.

\begin{tabular}{|l|l|l|l|l|l|}
\hline Element & $\begin{array}{l}\text { Detection } \\
\text { Limit (ppm) }\end{array}$ & $\begin{array}{l}\text { AOAP } \\
\text { Calibration } \\
(\mathbf{p p m})\end{array}$ & Element & $\begin{array}{l}\text { Detection } \\
\text { Limit (ppm) }\end{array}$ & $\begin{array}{l}\text { AOAP } \\
\text { Calibration } \\
(\mathbf{p p m})\end{array}$ \\
\hline Aluminum & $\mathrm{ns} *$ & 10 & Nickel & $5-10$ & 10 \\
\hline Boron & $\mathrm{ns}$ & 100 & Silicon & $\mathrm{ns}$ & 10 \\
\hline Chromium & $1-3$ & 10 & Silver** & $1-3$ & 10 \\
\hline Copper & $5-10$ & 10 & Sodium & $\mathrm{ns}$ & 10 \\
\hline Iron & $1-3$ & 10 & Tin & 10 & 10 \\
\hline Lead & $1-3$ & Titanium & $3-5$ & 10 \\
\hline Magnesium & $\mathrm{ns}$ & Zinc & $1-3$ & 100 \\
\hline Molybdenum & $3-5$ & 10 & & \\
\hline$*$ ns = Not in standard configuration of XRF. & & & \\
\hline ** Requires ${ }^{241}$ Americium source. & & & \\
\hline
\end{tabular}

Electromechanical viscometer. The standard ranges for the viscometer are viscosities from 2.5 to $50 \mathrm{cP}$ and from 25 to $500 \mathrm{cP}$ (other ranges available) with a repeatability of 0.1 percent. The AOAP calibration requirement is 6.75 to $430 \mathrm{cP}$.

Laser extinction particle counter. Can monitor particle size in up to 8 ranges between 4 and 100 microns. Is calibrated to ISO 11171:2000 standards (4, 6 and 14 microns).

Debris monitor. Monitors Ferrous and Non-ferrous wear particles. Minimum Ferrous particle size is 70 microns, minimum Non-ferrous particle size is 280 microns.

\section{Notes:}

1. It is important to keep in mind that this system was not designed to completely replace laboratory oil analysis. Its main purpose is to allow the user to determine several key parameters in the field in order to assess the condition of the oil on a near real time basis. This may provide adequate analysis in itself or serve as a screening mechanism to determine which samples, if any, require more detailed study.

2. AutOLab is a trademark of Belhaven Applied Technologies, Inc. 
Attachment 2

Reference Task 3: System Design

Attach 2 


\section{AutoSampler PCA System}

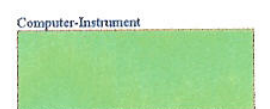

DWG No. E-0110-004-0
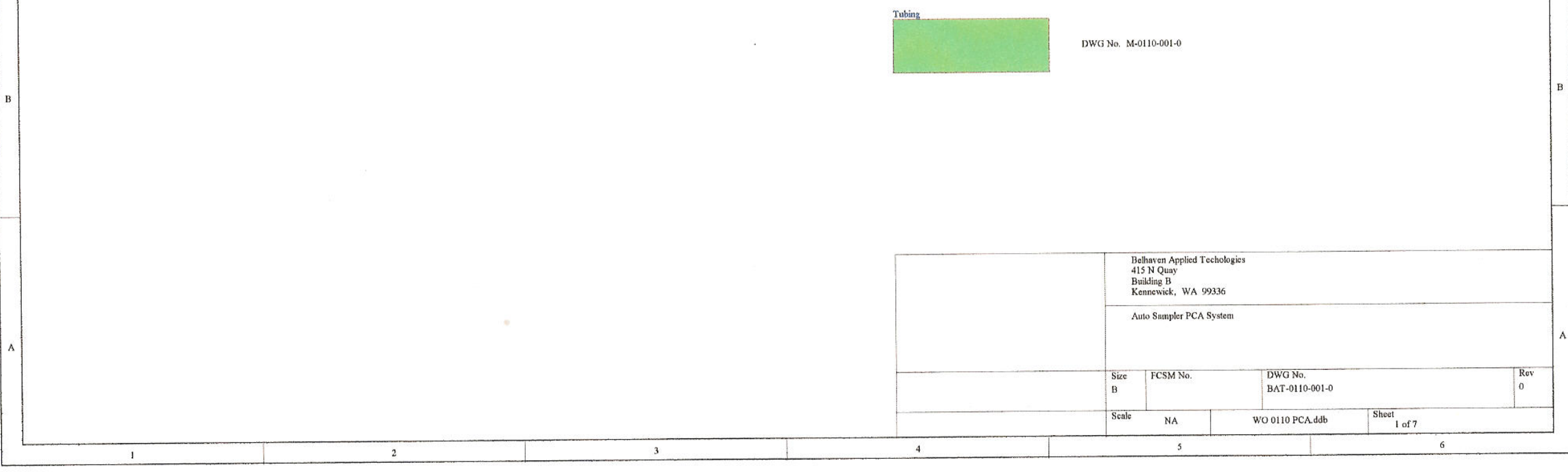


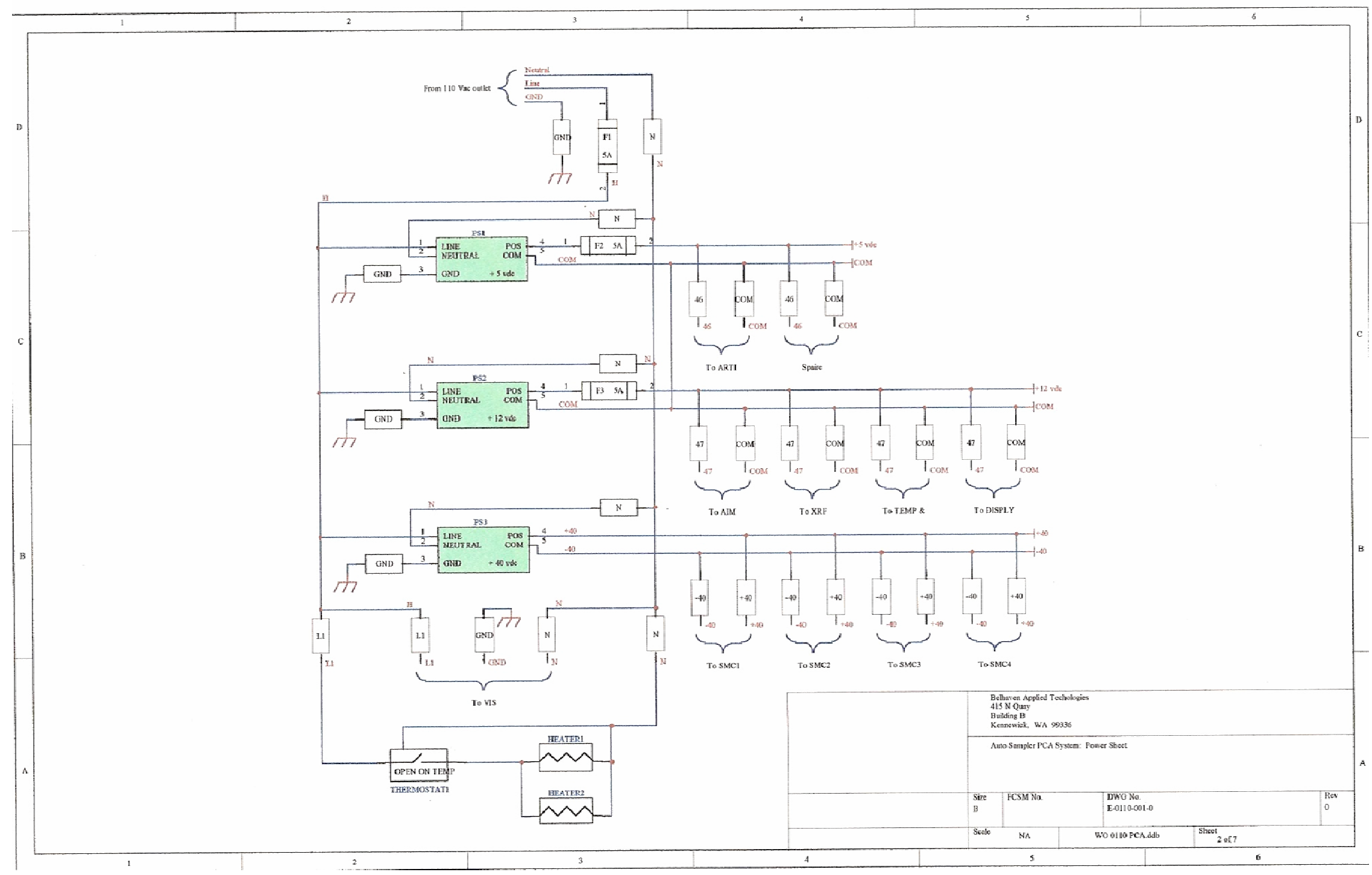




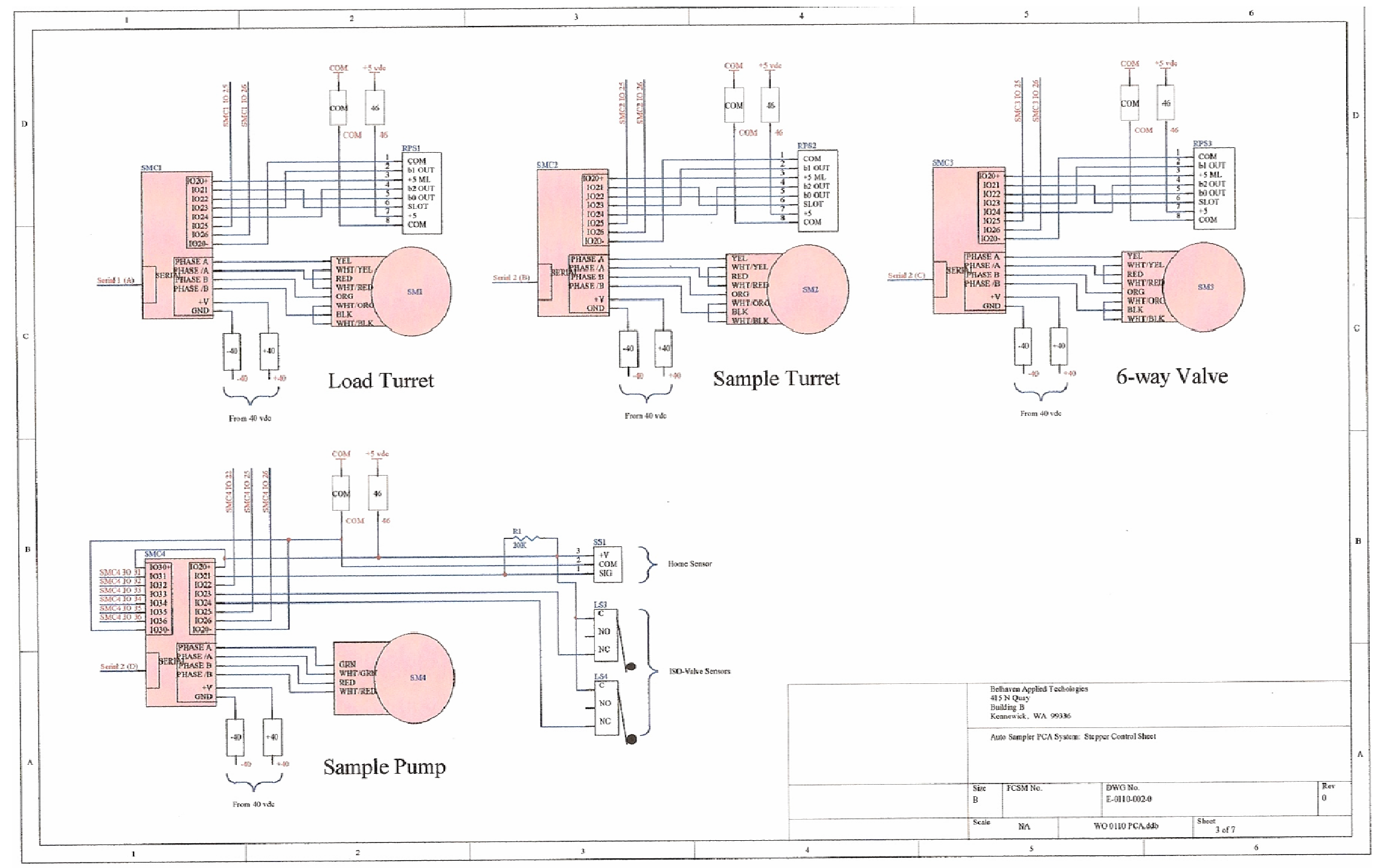




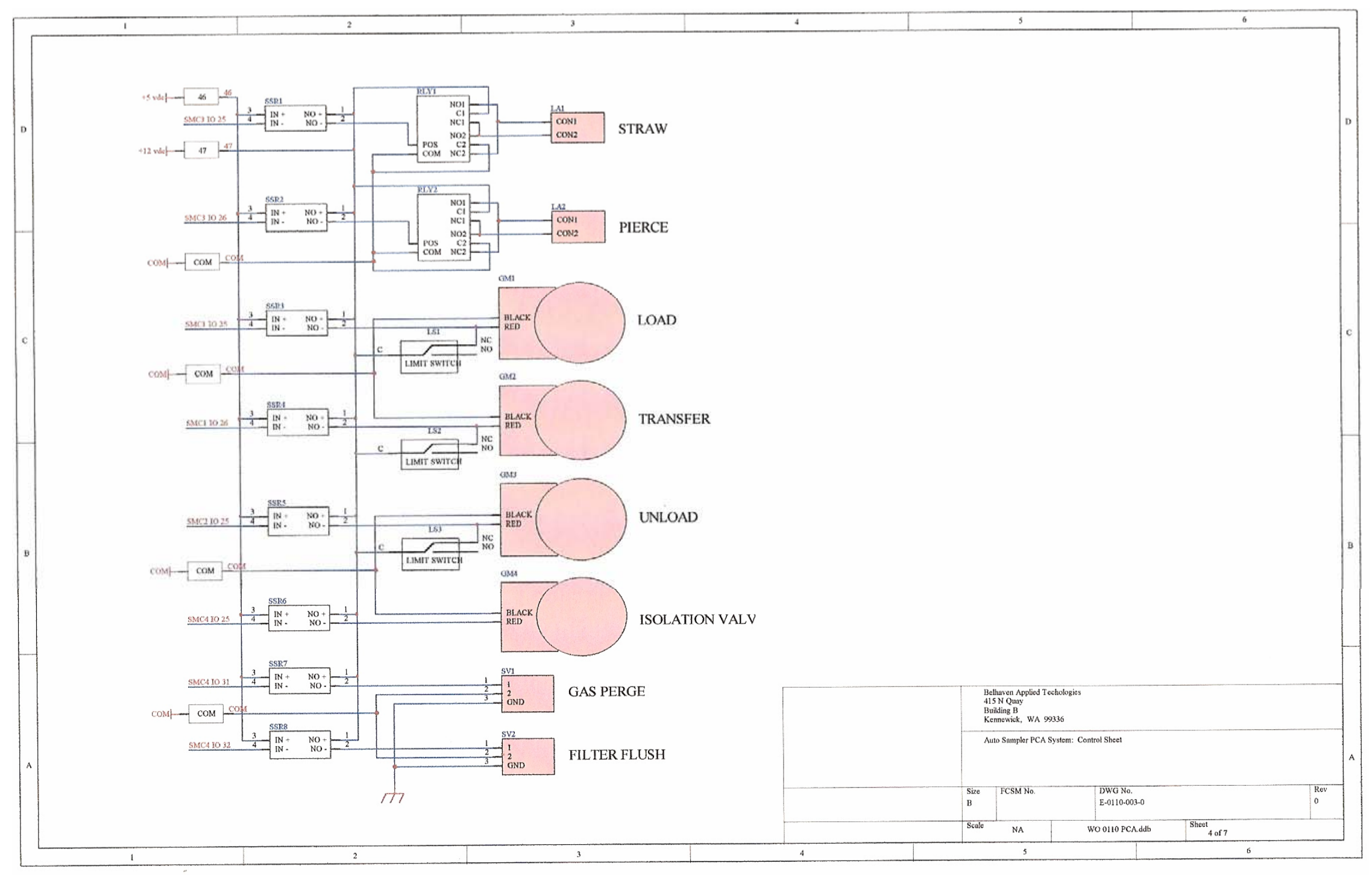




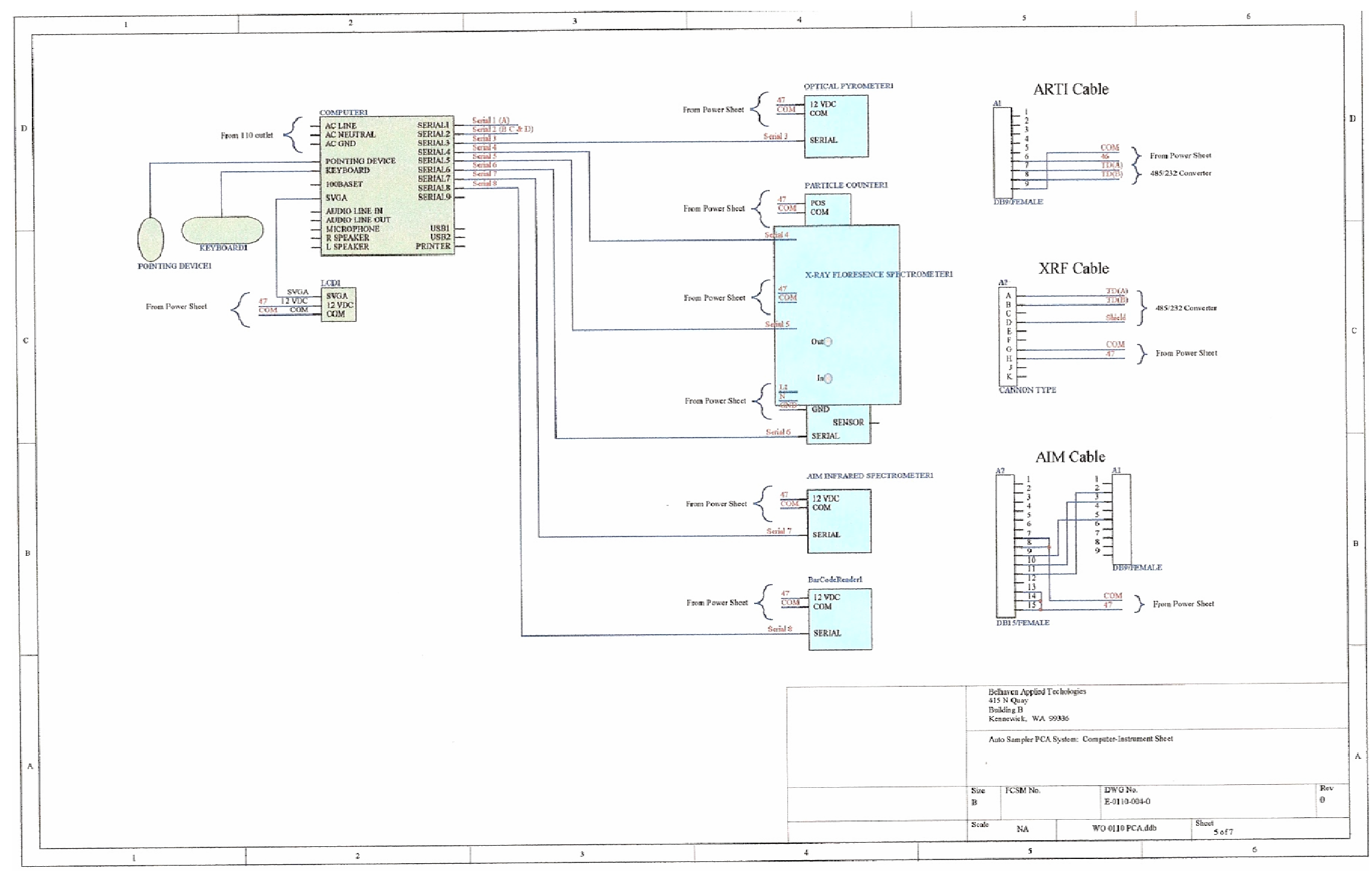




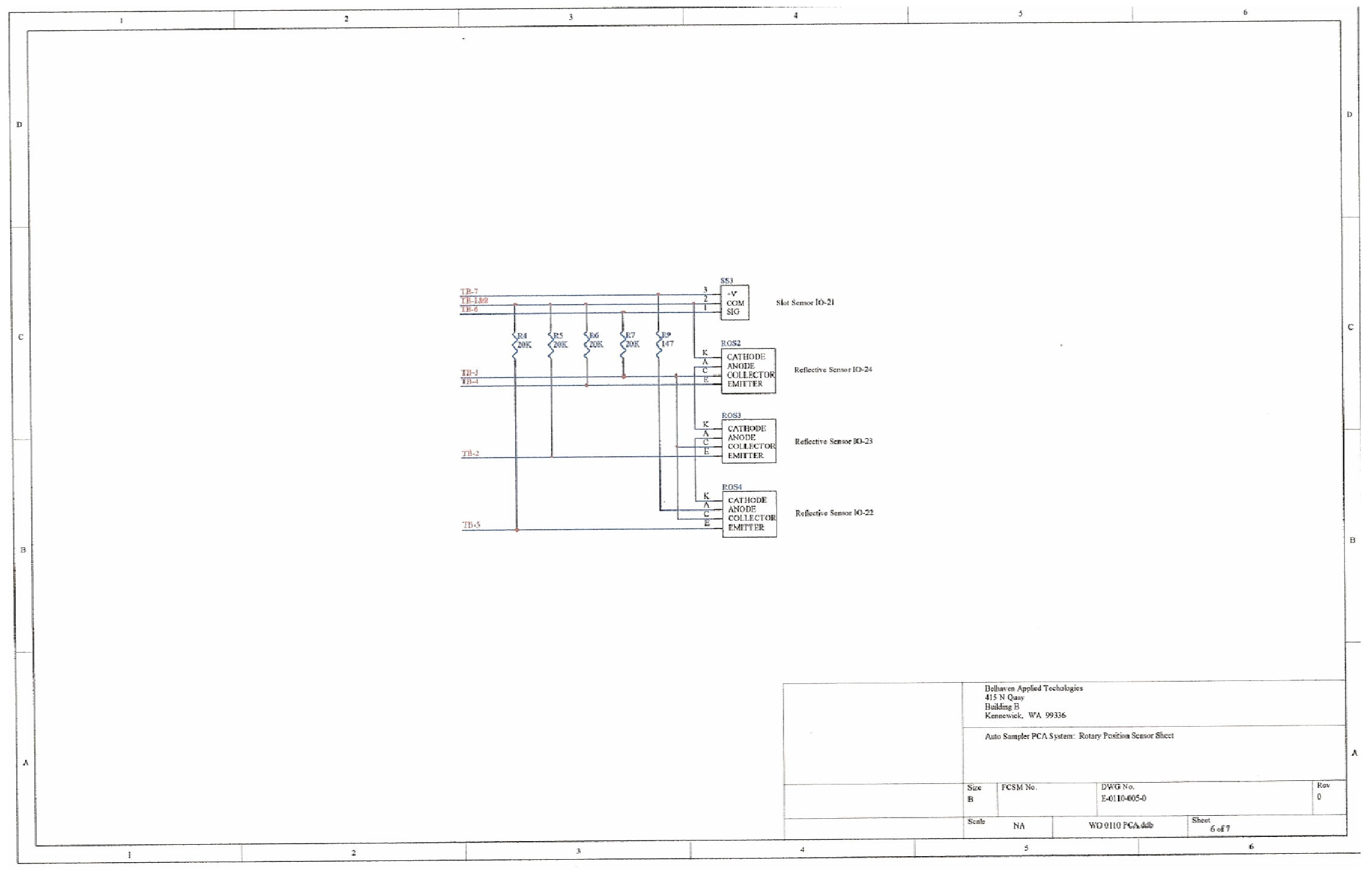




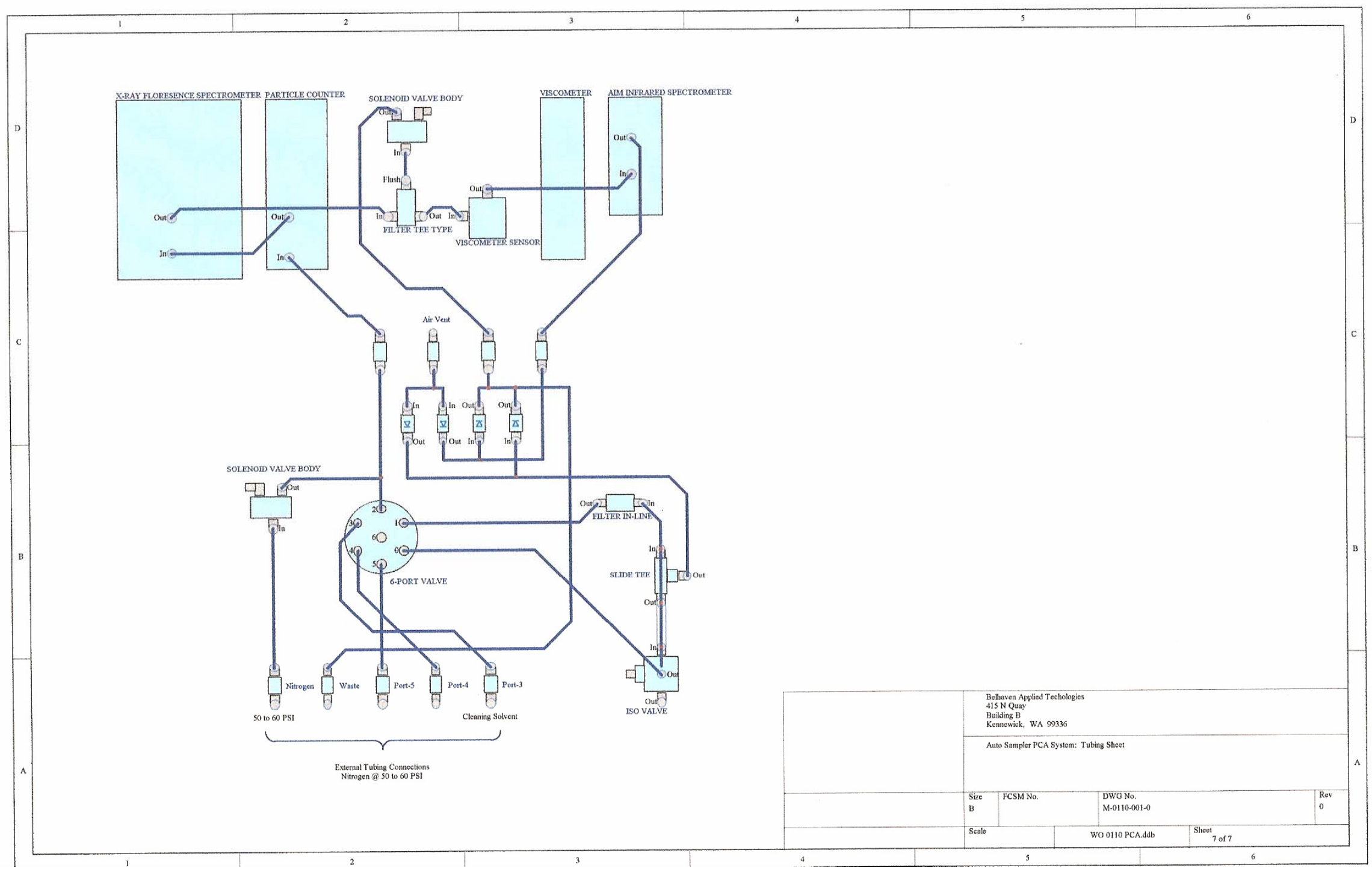




\section{Attachment 3}

Reference Task 5: Assembly, software modification and bench testing 


\section{Factory Acceptance Testing Requirements for Belhaven Lab-in-a-Box Prototype Unit 2.}

The purpose of this document is to specify the Factory Acceptance Test (FAT) that the second prototype Lab-in-a-Box (LIAB) must pass in order to fulfill the FAT requirements of Task 4 of PO 4016 issued by Battelle to Belhaven Applied Technologies. The FAT will 1) demonstrate the functionality of the hardware, 2) demonstrate that the instruments installed in the system operate and 3) insure that any changes made to the system between completion of 1 and 2 above and delivery do not affect the results of 1 and 2 above. The specifics of each of these requirements are spelled out below.

1. Functionality. Fulfillment of this requirement will demonstrate that the mechanical hardware of the AutoSampler is functional. The requirement is that at least 8 sample bottles run through the AutoSampler test cycle uninterrupted. These samples can be any samples that Belhaven wishes to use for this test. The system must load, register the bar code, condition the sample to a specified temperature (a hold at room temperature while the sample stirs is acceptable), run the sample through a test cycle, write test results in a spreadsheet readable format and clean itself between specimens using a clean cycle specified by Belhaven. There are no requirements on the values of the results written to the spreadsheet file for this test. If the system fails to complete the required tests, changes can be made to the system and the test may be repeated as many times as it takes to meet this requirement.

Operation of Instruments. Fulfillment of this requirement will demonstrate that the instruments in the LIAB operate. The requirement is that 5 oil samples supplied by Shell be run through the LIAB and that the results of these tests be within the ranges outlined below. These samples consist of 5 fluids of different viscosities.

The results from this sample testing must fall within the following ranges:

a. Viscosity: the temperature adjusted viscosity must fall within -10 percent of the low value of the sample's known viscosity range and +10 percent of the high value.

b. Particle counter: the result of the particle must fall within \pm 20 percent of the known values for the calibrated specimen.

c. XRF: the concentration of the zinc measured by the XRF must be within \pm 10 percent of the known concentration.

d. NDIR: the results must demonstrate that there is a relative agreement between the IR spectra provided by Shell for the oils being used in this test and the NDIR at the wavenumbers of the filters used in the NDIR.

The results will be considered acceptable if they fulfill one of the following requirements: 1) The absolute value of the results for the instrument fall within the ranges specified above or 2) it can be demonstrated that there is a repeatable bias in the test results and if this bias is taken into account the results fall within the ranges specified above. If the first of the options stated above is used, only 1 specimen needs to be run for the specified instrument. If the second option is used, a minimum of 3 specimens needs to be run for the specified instrument to demonstrate the repeatability of the results. 
2. Change Requirements. This requirement is to insure that any changes made to major components to the system between FAT and delivery does not affect the ability of the system to fulfill requirements 1 and 2 above. Major changes are considered to consist of replacement of any of the instruments, changes to the AutoSampler hardware, and changes in computer hardware and/or software. If any changes are made to these systems than it must be demonstrated that the affected systems still meet requirements 1 and 2 above.

Battelle will consider the FAT to be completed when it has received written notification from Belhaven that the testing is completed and documentation of the results. This documentation of results will consist of a brief description of the test procedures plus printed and electronic copies of the files generated by the LIAB during the testing. Battelle would like the option of observing any portion of this testing at Belhaven. 


\section{Pacific Northwest \\ National Laboratory \\ Operated by Battelle for the \\ U.S. Department of Energy}

May 11, 2004

Gene Silvernail

Belhaven Applied Technologies, Inc.

415 N Quay

Kennewick, WA 99336

Dear Gene:

This letter is to inform you that we have reviewed the results of the Factory Acceptance Testing and find them satisfactory. We consider Belhaven to have fulfilled the requirements of this portion of PO 4016 issued by Battelle. Attached are a summary of the results of this testing.

Sincerely,

pobetistrencenis

Bob Francini

Senior Research Scientist

Pacific Northwest National Laboratory

902 Battelle Boulevard • P.O. Box 999 • Richland, WA 99352

Telephone (509) 375-2193 ㅌ Email robert.francini@pnl.gov $\square$ Fax (509) 375-6497 
PCA Unit Acceptance Testing Summary

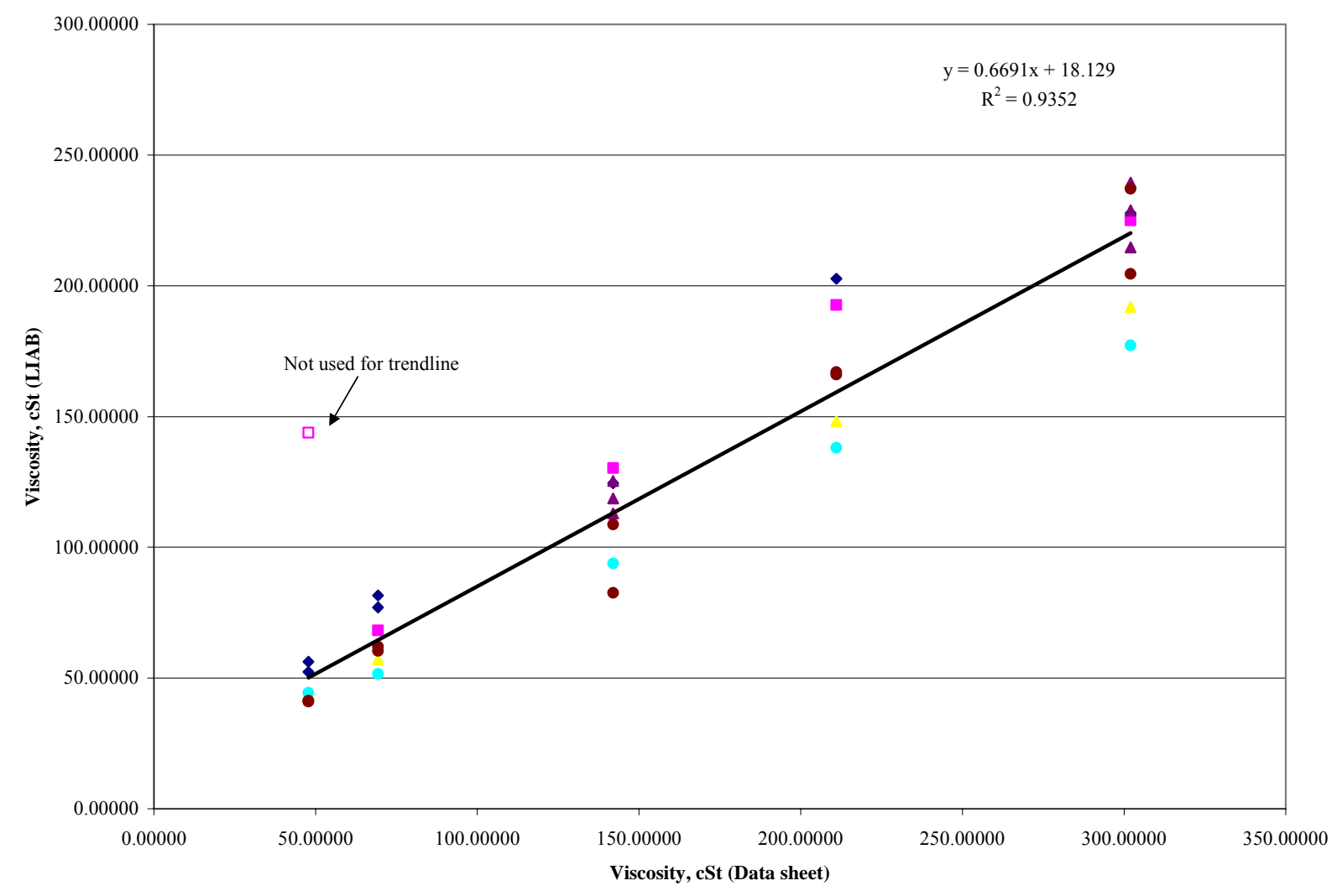


Tellus Plus 46

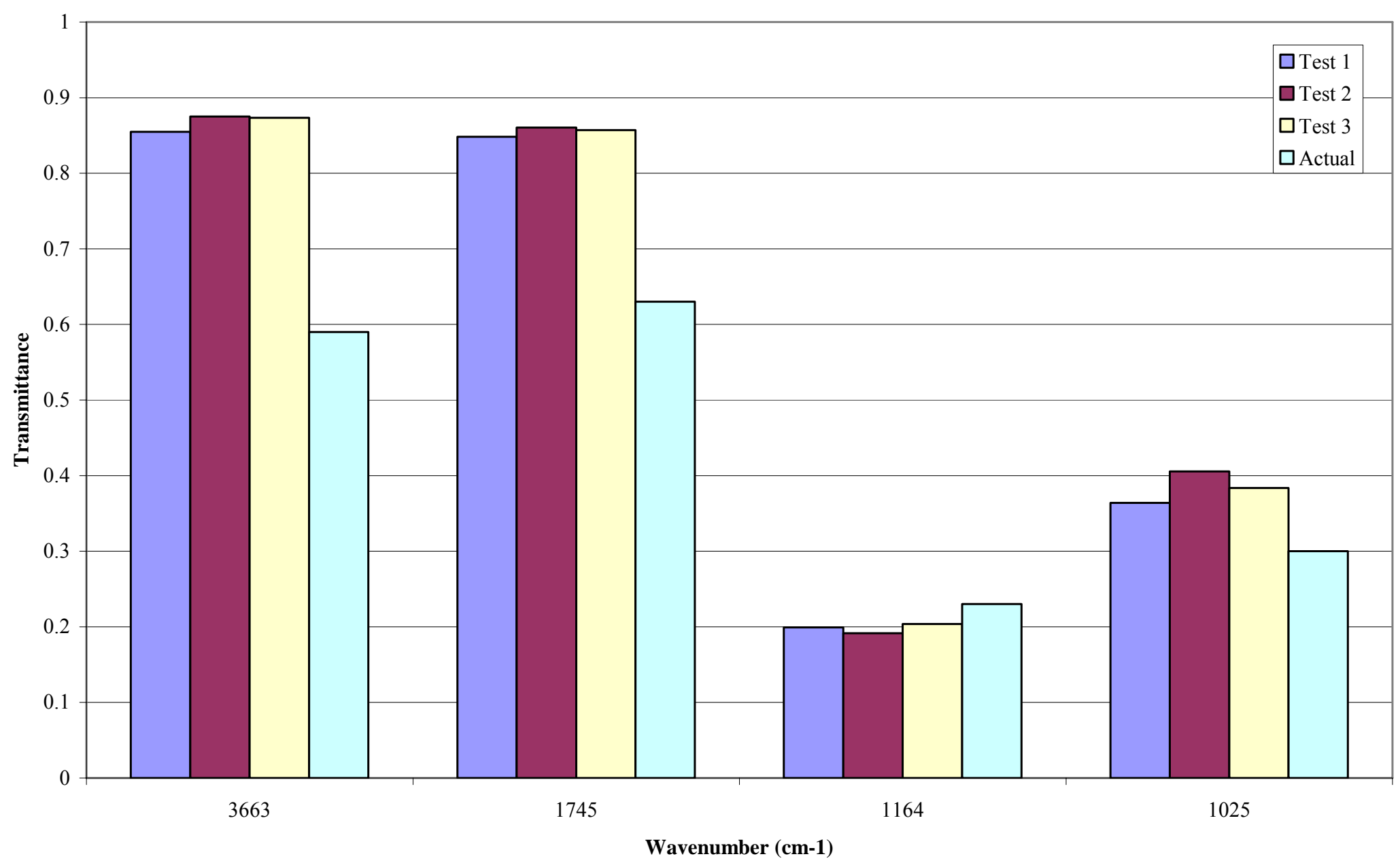


Turbo T 68

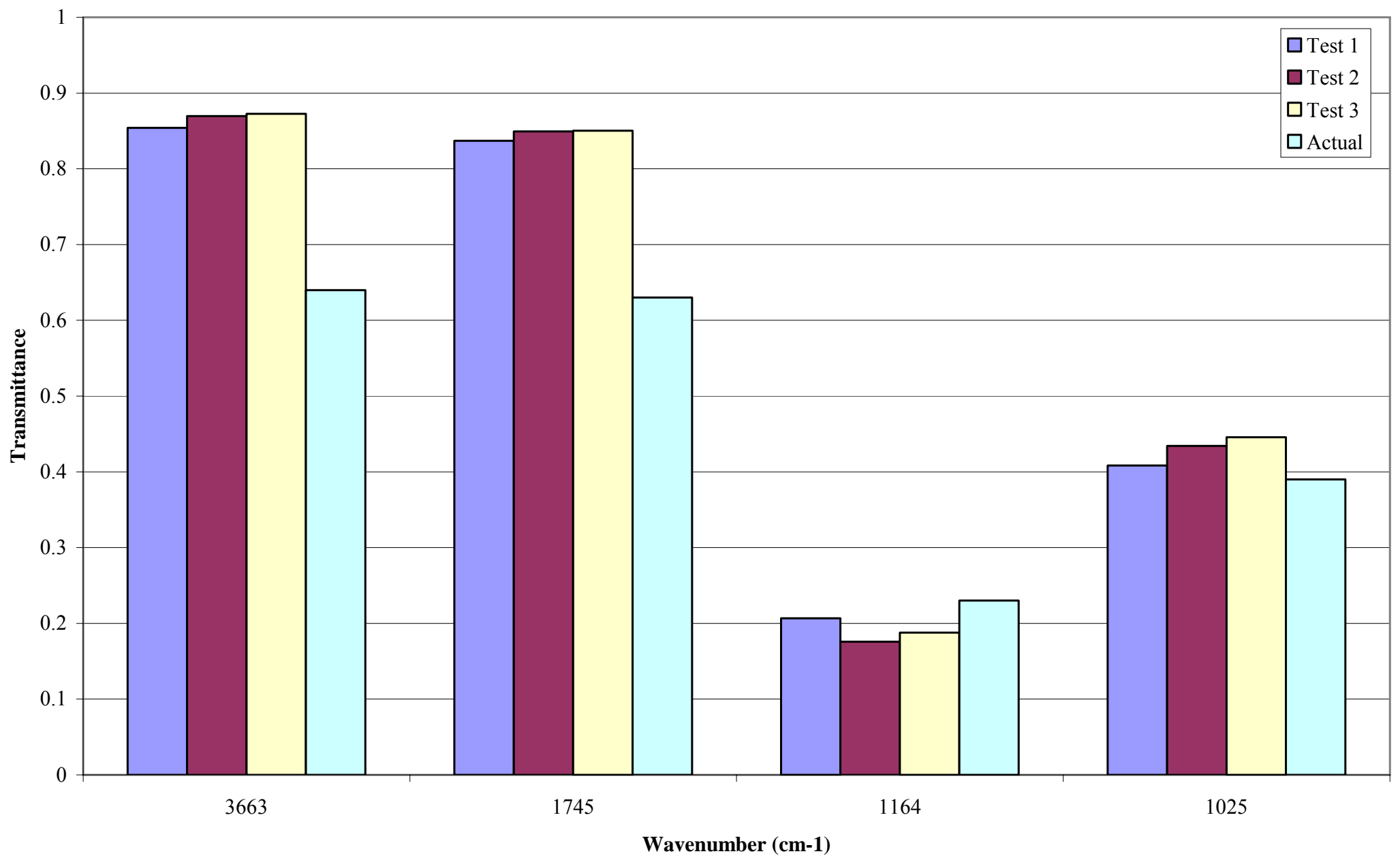


Tellus Plus 150

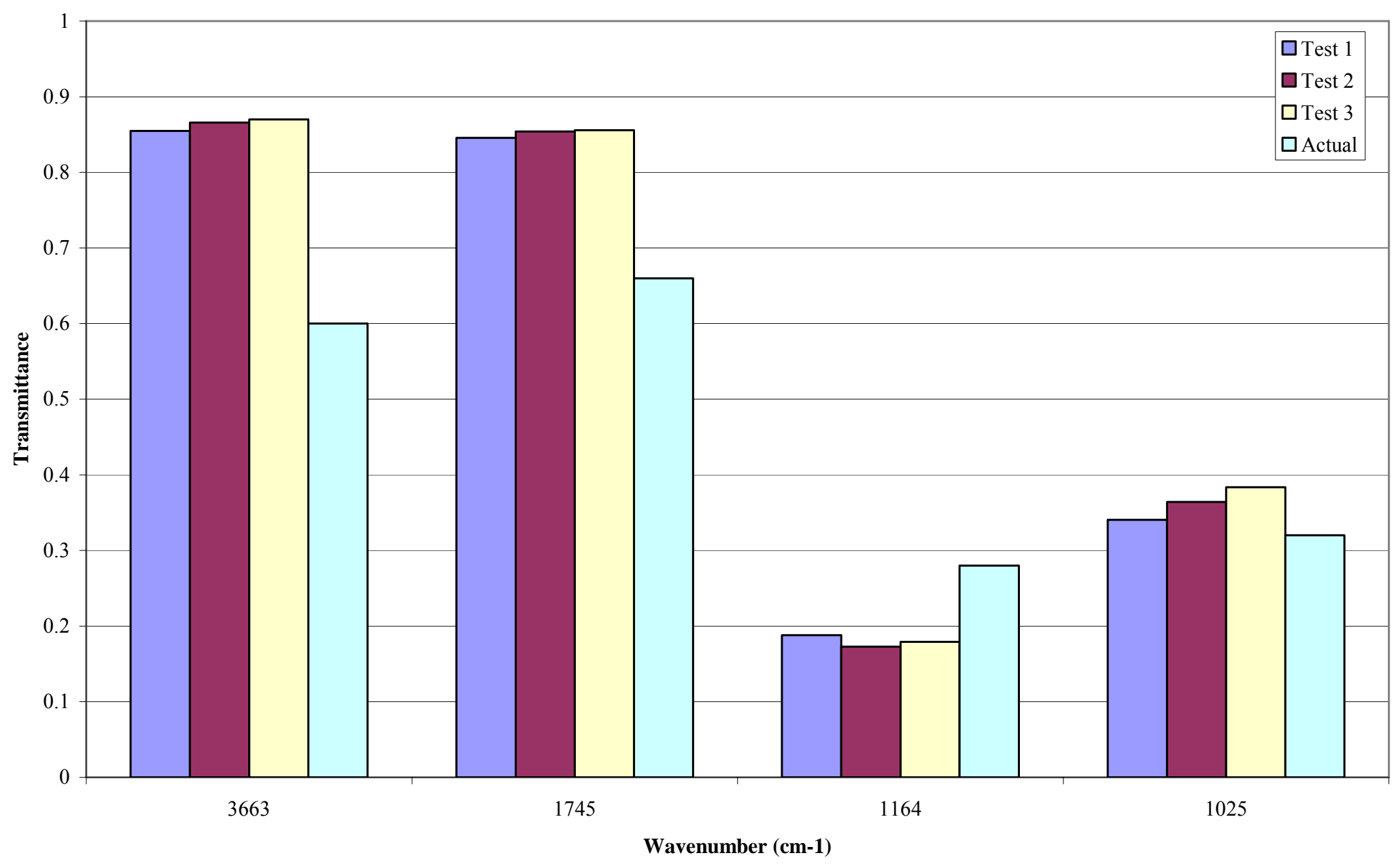


Delima EP 220

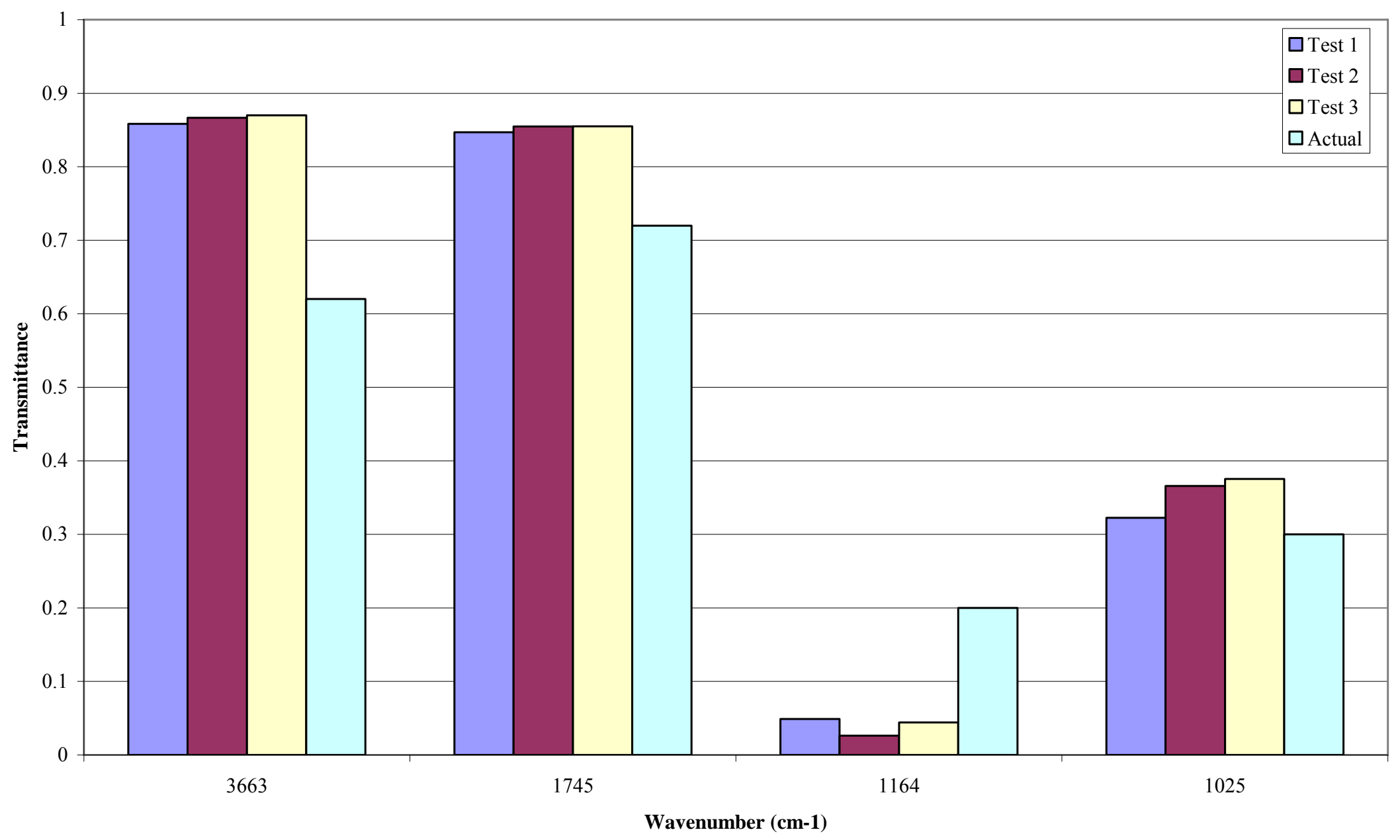


Omala 320

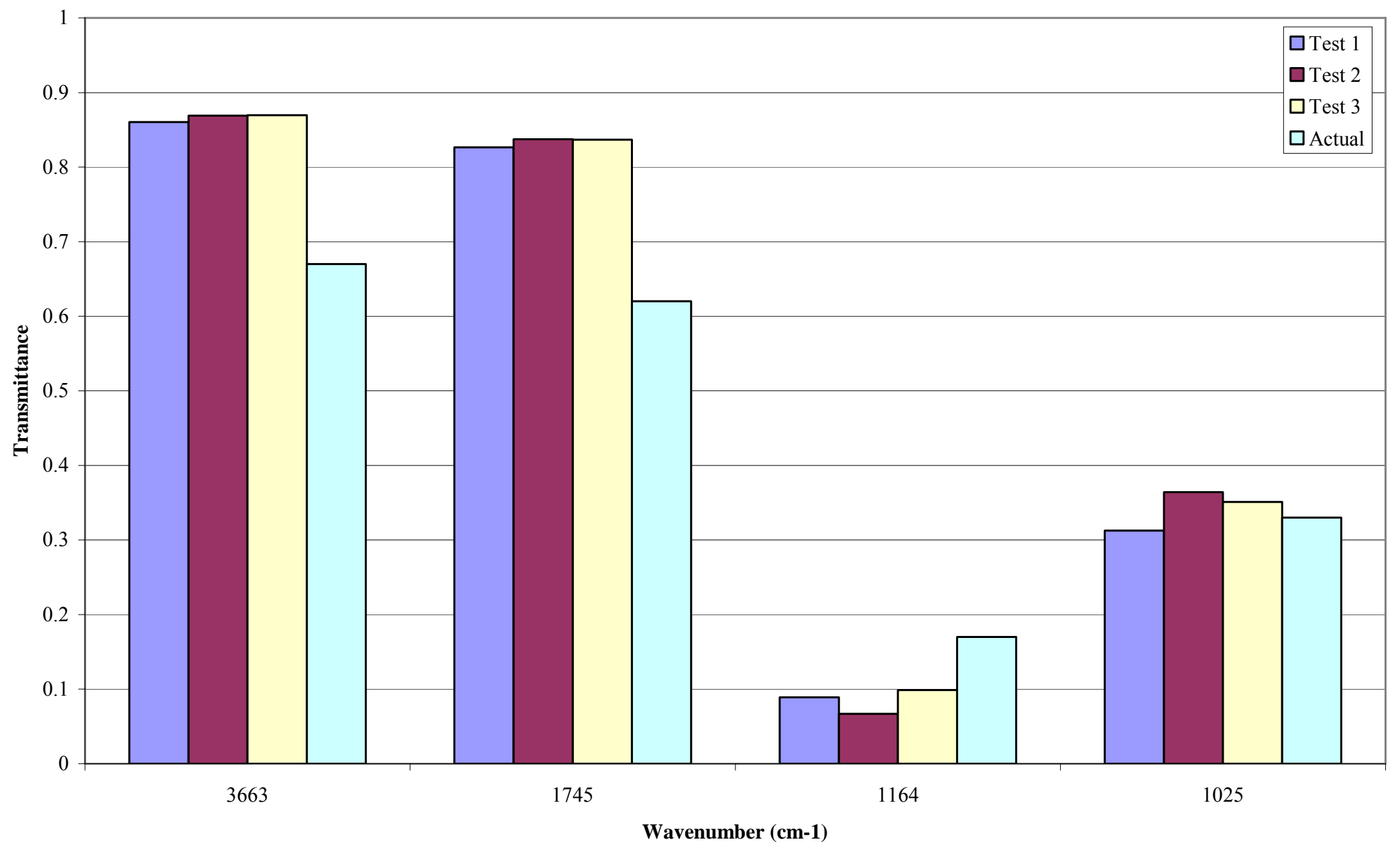




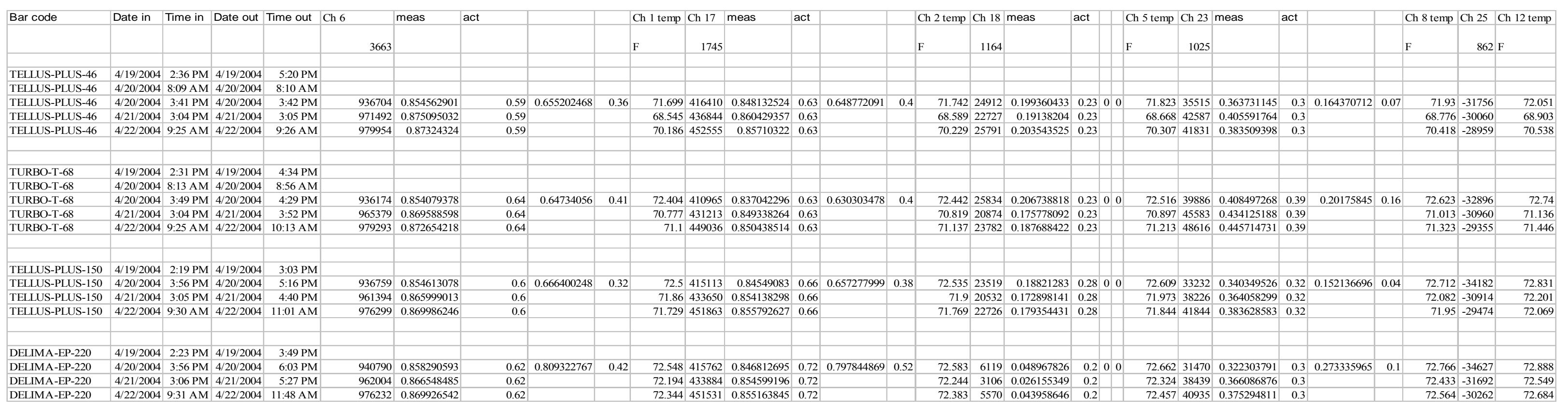




\begin{tabular}{|c|c|c|c|c|c|c|c|c|c|c|c|c|c|c|}
\hline Bar code & Date in & Time in & Date out & Time out & $\begin{array}{l}\text { Titanium } \\
\text { ppm }\end{array}$ & Vandium & Chromium & Iron & Nickel & Copper & Zinc & Zinc act & Lead & \begin{tabular}{|l|} 
Molybeden \\
ppm
\end{tabular} \\
\hline & & & & & ppm & ppm & ppm & ppm & ppm & ppm & ppm & ppm & ppm & \\
\hline TELLUS-PLUS-46 & 4/19/2004 & 2:36 PM & 4/19/2004 & 5:20 PM & 0 & 56 & 0 & 0 & 0 & 0 & 368 & 394 & 0 & 0 \\
\hline TELLUS-PLUS-46 & $4 / 20 / 2004$ & 8:09 AM & $4 / 20 / 2004$ & $8: 10$ AM & $\mathrm{O}$ & 70 & 0 & 0 & 0 & 0 & 368 & & $\mathrm{O}$ & 0 \\
\hline TELLUS-PLUS-46 & $4 / 20 / 2004$ & 3:41 PM & $4 / 20 / 2004$ & 3:42 PM & $\mathrm{O}$ & 38 & 0 & 0 & $\mathrm{o}$ & 0 & 321 & & 0 & 0 \\
\hline TELLUS-PLUS-46 & $4 / 21 / 2004$ & 3:04 PM & 4/21/2004 & 3:05 PM & 0 & 1 & 0 & 0 & 0 & 0 & 362 & & 0 & 0 \\
\hline TELLUS-PLUS-46 & 4/22/2004 & $9: 25$ AM & $4 / 22 / 2004$ & $9: 26$ AM & $\mathrm{o}$ & 3 & 0 & 0 & $\mathrm{o}$ & 0 & 360 & & 0 & 0 \\
\hline & & & & & & & & & & & & & & \\
\hline TURBO-T-68 & 4/19/2004 & 2:31 PM & 4/19/2004 & 4:34 PM & 0 & 0 & 0 & 0 & 0 & 0 & 0 & 2 & 0 & 0 \\
\hline TURBO-T-68 & $4 / 20 / 2004$ & 8:13 AM & $4 / 20 / 2004$ & 8:56 AM & 0 & 0 & 0 & 0 & 0 & 0 & 12 & & 2 & 1 \\
\hline TURBO-T-68 & $4 / 20 / 2004$ & 3:49 PM & $4 / 20 / 2004$ & 4:29 PM & $\mathrm{O}$ & 0 & 0 & 0 & 0 & 0 & 0 & & 0 & 0 \\
\hline TURBO-T-68 & $4 / 21 / 2004$ & 3:04 PM & $4 / 21 / 2004$ & 3:52 PM & 0 & 0 & 0 & 0 & 0 & 0 & 0 & & 2 & 0 \\
\hline TURBO-T-68 & $4 / 22 / 2004$ & 9:25 AM & 4/22/2004 & 10:13 AM & 0 & 6 & 0 & 0 & 0 & 0 & 0 & & 2 & 1 \\
\hline & & & & & & & & & & & & & - & \\
\hline TELLUS-PLUS-150 & 4/19/2004 & 2:19 PM & 4/19/2004 & 3:03 PM & 0 & 30 & 0 & 0 & 0 & 0 & 323 & 318 & 0 & 1 \\
\hline TELLUS-PLUS-150 & $4 / 20 / 2004$ & 3:56 PM & $4 / 20 / 2004$ & 5:16 PM & $\mathrm{O}$ & 9 & $\mathrm{O}$ & 0 & $\mathrm{O}$ & $\mathrm{O}$ & 323 & & $\mathrm{O}$ & 1 \\
\hline TELLUS-PLUS-150 & 4/21/2004 & 3:05 PM & 4/21/2004 & 4:40 PM & 0 & 37 & 0 & 0 & 0 & 0 & 318 & & 0 & 0 \\
\hline TELLUS-PLUS-150 & 4/22/2004 & 9:30 AM & 4/22/2004 & 11:01 AM & 0 & 19 & 0 & 0 & 0 & 0 & 314 & & 0 & 1 \\
\hline & & & & & & & & & & & & & - & \\
\hline DELIMA-EP-220 & 4/19/2004 & 2:23 PM & 4/19/2004 & 3:49 PM & 0 & 0 & 0 & 0 & 0 & 0 & 0 & 3 & 0 & 1 \\
\hline DELIMA-EP-220 & $4 / 20 / 2004$ & 3:56 PM & $4 / 20 / 2004$ & 6:03 PM & 0 & 0 & 0 & 0 & 0 & 0 & 0 & & 0 & 1 \\
\hline DELIMA-EP-220 & $4 / 21 / 2004$ & 3:06 PM & $4 / 21 / 2004$ & 5:27 PM & 0 & 0 & 0 & 0 & $\mathrm{O}$ & 0 & 0 & & 0 & 1 \\
\hline DELIMA-EP-220 & $4 / 22 / 2004$ & 9:31 AM & $4 / 22 / 2004$ & 11:48 AM & 0 & 0 & 0 & 0 & 0 & 0 & 0 & & 0 & 0 \\
\hline & & & & & & & & & & & & & & \\
\hline OMALA-320 & 4/19/2004 & 2:17 PM & 4/19/2004 & 2:18 PM & 0 & 0 & 3 & 0 & 0 & 0 & 0 & 2 & 0 & 1 \\
\hline OMALA-320 & $4 / 20 / 2004$ & 3:57 PM & $4 / 20 / 2004$ & 6:50 PM & $\mathrm{O}$ & 6 & 0 & 0 & 0 & 0 & 0 & & 0 & 1 \\
\hline OMALA-320 & $4 / 21 / 2004$ & 3:06 PM & $4 / 21 / 2004$ & 6:14 PM & 0 & 0 & 0 & 0 & $\mathrm{O}$ & $\mathrm{o}$ & $\mathrm{o}$ & & 0 & 0 \\
\hline OMALA-320 & 4/22/2004 & 9:32 AM & $4 / 22 / 2004$ & 12:35 PM & 0 & 0 & 0 & 0 & 0 & 0 & 0 & & 0 & 0 \\
\hline & & & & & & & & & & & & & & \\
\hline TELLUS-PLUS-150 & $4 / 27 / 2004$ & 2:32 PM & $4 / 27 / 2004$ & 2:33 PM & 0 & 64 & 0 & 0 & 0 & 0 & 319 & 318 & 1 & $\mathrm{O}$ \\
\hline TELLUS-PLUS-150 & $4 / 27 / 2004$ & 2:32 PM & $4 / 27 / 2004$ & 3:20 PM & 0 & 28 & 0 & 0 & 0 & 0 & 320 & & 1 & 1 \\
\hline TELLUS-PLUS-150 & $4 / 27 / 2004$ & 2:33 PM & $4 / 27 / 2004$ & 4:07 PM & 0 & 48 & 0 & 0 & $\mathrm{O}$ & 0 & 317 & & 0 & 1 \\
\hline OMALA-320 & $4 / 27 / 2004$ & 2:35 PM & $4 / 27 / 2004$ & 4:54 PM & 0 & 0 & 0 & 0 & 0 & 0 & 0 & 2 & 1 & 1 \\
\hline OMALA-320 & 4/27/2004 & 2:41 PM & $4 / 27 / 2004$ & 5:41 PM & 0 & 0 & 0 & 0 & 0 & 0 & 0 & & 0 & 0 \\
\hline OMALA-320 & $4 / 27 / 2004$ & 3:14 PM & $4 / 27 / 2004$ & 6:29 PM & 0 & 0 & 0 & 0 & $\mathrm{o}$ & 0 & 0 & & 0 & 1 \\
\hline & & & & & & & & & & & & & & \\
\hline TELLUS-PLUS-150 & $4 / 28 / 2004$ & 2:21 PM & $4 / 28 / 2004$ & 2:22 PM & 0 & 14 & 0 & 0 & 0 & 0 & 315 & 318 & 0 & 1 \\
\hline TELLUS-PLUS-150 & $4 / 28 / 2004$ & 2:22 PM & $4 / 28 / 2004$ & 3:12 PM & 0 & 26 & 0 & 0 & $\mathrm{O}$ & 0 & 326 & 318 & 1 & 0 \\
\hline OMALA-320 & $4 / 28 / 2004$ & 2:24 PM & $4 / 28 / 2004$ & 3:59 PM & $\mathrm{O}$ & 22 & 0 & 0 & $\mathrm{O}$ & $\mathrm{o}$ & 0 & 2 & 0 & 1 \\
\hline OMALA-320 & $4 / 28 / 2004$ & 2:29 PM & $4 / 28 / 2004$ & 4:46 PM & 0 & 0 & 0 & 0 & 0 & 0 & 0 & 2 & 0 & 0 \\
\hline DELIMA-EP-220 & $4 / 28 / 2004$ & 2:29 PM & $4 / 28 / 2004$ & 5:33 PM & $\mathrm{O}$ & 6 & 0 & 0 & $\mathrm{O}$ & 0 & 0 & 3 & 0 & 1 \\
\hline DELIMA-EP-220 & $4 / 28 / 2004$ & 2:38 PM & $4 / 28 / 2004$ & 6:20 PM & 0 & 0 & 0 & 0 & $\mathrm{o}$ & 0 & 0 & 3 & 0 & 1 \\
\hline TURBO-T-68 & $4 / 28 / 2004$ & 2:42 PM & $4 / 28 / 2004$ & 7:08 PM & 0 & 0 & 11 & 0 & 0 & 0 & 0 & 2 & 0 & 1 \\
\hline TURBO-T-68 & $4 / 28 / 2004$ & 2:43 PM & $4 / 28 / 2004$ & 7:55 PM & $\mathrm{O}$ & 11 & 0 & 0 & $\mathrm{o}$ & $\mathrm{o}$ & 0 & 2 & 0 & 0 \\
\hline TELLUS-PLUS-46 & $4 / 28 / 2004$ & 2:44 PM & $4 / 28 / 2004$ & 8:42 PM & $\mathrm{O}$ & 14 & 0 & 0 & 0 & $\mathrm{o}$ & 367 & 394 & 0 & $\mathrm{O}$ \\
\hline TELLUS-PLUS-46 & $4 / 28 / 2004$ & 3:12 PM & $4 / 28 / 2004$ & 9:29 PM & $\mathrm{O}$ & 57 & 0 & 0 & $\mathrm{O}$ & 0 & 374 & 394 & 0 & 0 \\
\hline
\end{tabular}


Bar code Date in Time in Date out Time out 30 second particle coun

50 5/15 count Actan 1so sist

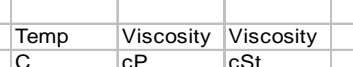
Temp comp Measured
Viscosity Viscosity
cemp Visco

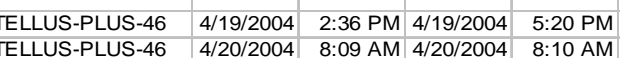

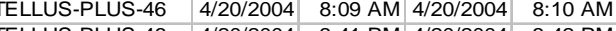
TELLUS-PLUS-46

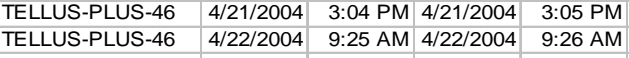

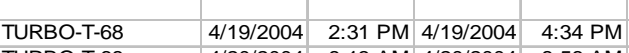
$\begin{array}{llllll} & \text { TURBO-T-68 } & \text { 4/20/2004 } & 8: 13 \text { AM 4/20/2004 } & 8: 56 \text { AM }\end{array}$ TURBO-T-68 4 T21/2004 3.04 PM 4/21/2004 3.25 PM TURBO-T-68
TURBO-T-68

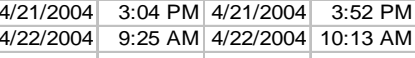

$\begin{array}{lllll}\text { TELLUS-PLUS-150 } & \text { 4/19/2004 } & \text { 2:19 PM 4/19/2004 } & \text { 3:03 PM }\end{array}$ $\begin{array}{lllll} & & & \\ \text { TELLUS-PLUS-150 } & 4 / 120 / 2004 & \text { 2:19 PM 4/19/2004 } & \text { 3:03 PM } \\ & \text { 3:56 PM 4/20/2004 } & \text { 5:16 PM }\end{array}$ \begin{tabular}{l|lllllll} 
& &
\end{tabular}

(2)

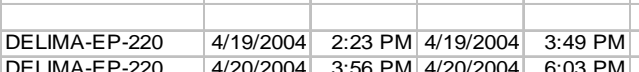
DELIMA-EP-220 4/20/2004 356 PM 4/20/2004 6:03 PM \begin{tabular}{lllll} 
DELIMA-EP-220 & $4 / 21 / 2004$ & $3: 06$ PM 4/21/2004 & 5:27 PM \\
\hline DELIMA-EP-220 & $4 / 22 / 2004$ & $9: 31$ AM 4/22/2004 & 11:48 AM
\end{tabular}

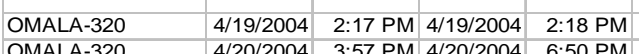

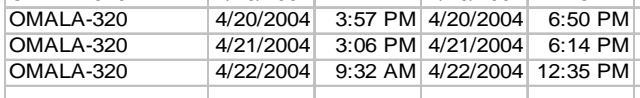

\begin{tabular}{|l|l|l|l}
1926 & 1075 & 363 & \\
\hline 2046 & 1286 & 432 & \\
\hline 3690 & 2306 & 716 & 1 \\
& & & \\
& & & \\
\hline 3057 & 1241 & 123 & \\
\hline 5000 & 24404 & 402 & \\
3994 & 1899 & 260 & \\
\hline 4813 & 2318 & 398 & \\
& & &
\end{tabular}

\begin{tabular}{|r|r|r|r|}
\hline 4009 & 2341 & 633 & 88 \\
\hline 3656 & 2154 & 579 & 93 \\
\hline 3891 & 2341 & 765 & 128 \\
\hline
\end{tabular}

\begin{tabular}{|r|r|}
\hline 707 & 368 \\
\hline 731 & 358 \\
\hline 2341 & 1349 \\
\hline
\end{tabular}

\begin{tabular}{|r|r|r|}
$2777 \quad 15$ \\
\hline
\end{tabular}

\begin{tabular}{r|r|}
2777 & 1570 \\
\hline 1590 & 942 \\
\hline 2203 & 1344 \\
\hline
\end{tabular} $\begin{array}{llllll} & & & \\ \text { TELLUS-PLUS-150 } & \text { 4/27/2004 } & \text { 2:32 PM 4/27/2004 } & \text { 2:33 PM off line } \\ \text { TELUS-PLUS-155 } & \text { 4/27/22004 } & 2: 32 \text { PM 4/27/2004 } & \text { 3:20 PM off line }\end{array}$

$\begin{array}{lllll}\text { TELLUS-PLUS-150 4/27/2004 } & \text { 2:33 PM 4/27/2004 4:07 PM off line }\end{array}$

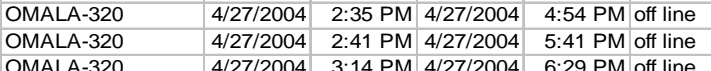

OMALA-320 4/27/2004 3:14 PM 4/27/2004 6:29 PM off

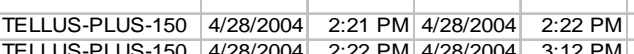
\begin{tabular}{llllll} 
TELLUS-PLUS-150 & $4 / 28 / 2004$ & $2: 22 \mathrm{PM}$ & $4 / 28 / 2004$ & $3: 12 \mathrm{PM}$ \\
OMALA-320 & $4 / 28 / 2004$ & $2 \cdot 24 \mathrm{PM}$ & $4 / 28 / 2004$ & $3: 59 \mathrm{PM}$ \\
\hline
\end{tabular}

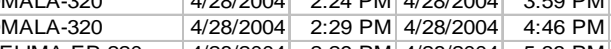

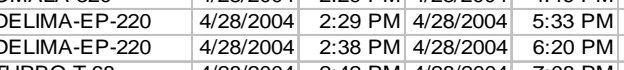
TURBO-T-68

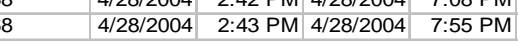
$\begin{array}{lllll}\text { TELLUS-PLUS-46 } & 4 / 22822004 & 2.44 \text { PM } & \text { 4/282/2004 } & \text { 8:42 PM } \\ \text { TELLUS-PLUS-46 } & 4 / 28 / 2004 & 3: 12 \text { PM 4/28/2004 } & 9: 29 \text { PM }\end{array}$

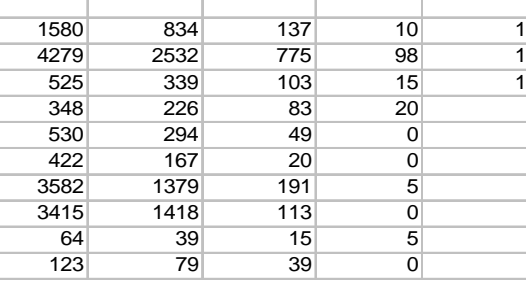

\begin{tabular}{|r|r|}
\hline 36 & 21 \\
\hline 25 & 5 \\
\hline 15 & 5 \\
\hline & \\
\hline 15 & 10 \\
\hline 25 & 10 \\
\hline 0 & 0 \\
\hline 10 & 0 \\
\hline
\end{tabular}

\begin{tabular}{|c|c|}
\hline & \\
0 & 0 \\
0 & 0 \\
\hline 0 & 0 \\
\hline & \\
\hline & \\
\hline 0 & 0 \\
\hline 0 & 0 \\
\hline & 0 \\
\hline
\end{tabular}

\begin{tabular}{|r|r|r|} 
& & \\
\hline 48200 & $210016 / 12$ & $14 / 10$ \\
53100 & 50016110 & 14110 \\
\hline 84900 & $50017 / 10$ & $14 / 10$ \\
\hline & & \\
\hline
\end{tabular}

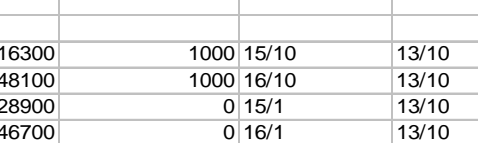
$\begin{array}{ll}0 & 15 / 1 \\ 0 & 16 / 1\end{array}$

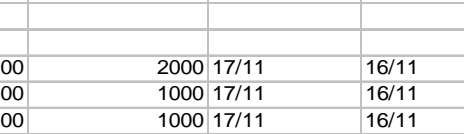

95200

13800
10300
41700 $100014 / 10$
5001410
$016 / 1$

\begin{tabular}{l|l|l}
\hline 59800 & $100016 / 10$ & $16 / 12$ \\
\hline 34800 & $100016 / 11$ & $16 / 12$ \\
\hline
\end{tabular}

(2.

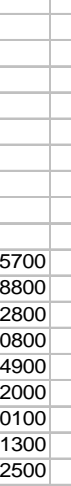
2500
3900 \begin{tabular}{|r|r|r|r|r|r|r|}
91.37 & 32.98333 & 61.209 & 68.77416 & -0.00610 & 52.29948 & 47.80000 \\
\hline 90.651 & 32.58389 & 67.122 & 75.41798 & -0.00610 & 56.25441 & \\
\hline 81.09 & 27.27222 & 230.31 & 258.7753 & -0.00610 & 143.83960 &
\end{tabular}

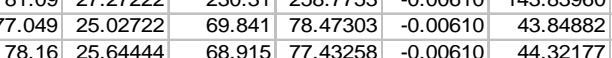

\begin{tabular}{l|l|l|l|l|l|l}
93.361 & 34.08944 & 93.019 & 104.5157 & -0.00615 & 81.53643 & 69.30000
\end{tabular}

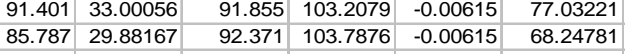
$\begin{array}{rrrrrr}79.124 & 26.18 & 92.354 & 100.3978 & -0.00615 & 57.15061 \\ 79.174 & 26.20778 & 79.782 & 89.6427 & -0.00615 & 51.55691\end{array}$

$\begin{array}{lllllllll}84.387 & 29.10389 & 184.098 & 206.8517 & -0.00631 & 124.52383 & 142.00000\end{array}$

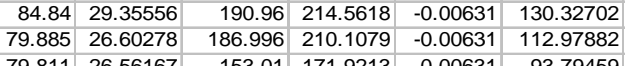

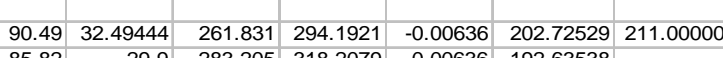
\begin{tabular}{rrrrrr}
85.82 & 29.9 & 283.205 & 318.2079 & -0.00636 & 192.63538 \\
80.181 & 26.76722 & 250.399 & 28.143472 & -0.00636 & 148.17333 \\
\hline 80.436 & 26.90889 & 230.29 & 258.7528 & -0.00636 & 138.09473
\end{tabular}

$\begin{array}{lllllll}82.649 & 28.13833 & 372.738 & 418.8067 & -0.00639 & 227.73544 & 302.00000\end{array}$ $\begin{array}{lllllll}84.224 & 29.01333 & 351.215 & 394.6236 & -0.00639 & 225.00482 \\ 80.378 & 26.87667 & 331.053 & 371.9697 & -0.00639 & 191.90711\end{array}$

$\begin{array}{llllll}80.378 & 26.87667 & 331.053 & 371.9697 & -0.00639 & 191.90711 \\ 80.812 & 27.11778 & 29.829 & 336.8865 & -0.00639 & 177.22928\end{array}$

$\begin{array}{rrrrrrr}79.451 & 26.36167 & 189.133 & 212.509 & -0.00631 & 112.94874 & 142.00000\end{array}$

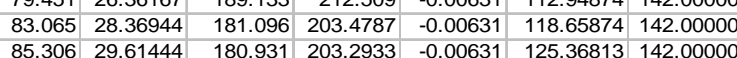

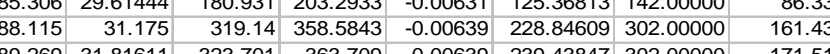
$\begin{array}{llllllll}89.269 & 31.81611 & 323.701 & 363.709 & -0.00069 & 239.43847 & 322.00000 & 171.51 \\ 85.571 & 29.76167 & 321.081 & 360.7652 & -0.00639 & 214.67566 & 302.00000 & 146.338\end{array}$ \begin{tabular}{rrrr|r|r|}
81.08 & 27.26667 & 128.86 & 144.7865 & -0.00631 & 82.52696 \\
142.00000
\end{tabular} $\begin{array}{rrrr}85.461 & 29.70056 & 154.733 & 173.8 \\ 86.89 & 30.49444 & 293597 & -329.8 \\ 8\end{array}$

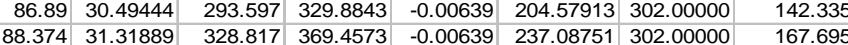

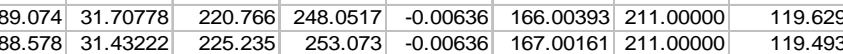

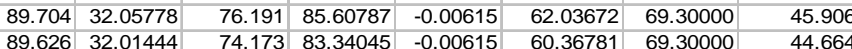

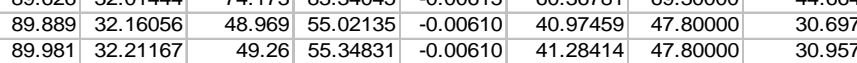




\section{Lab-in-a-Box Software Operating Manual PNNL-14821}

\section{Introduction}

The Lab-in-a-Box (LIAB) consists of four instruments commonly used in machine fluid analysis. These instruments are a viscometer, a particle counter, a XRF elemental analyzer and a Non-dispersive infrared analyzer. These four instruments are linked through a common flow path with an automatic sampling unit that holds up to 9 test specimens, including the sample being tested. The instruments and the sampling unit are controlled using a LabView software master program. All control is through serial port commands. The remainder of this manual summarizes the use of this master program. Information and specifications for the different instruments can be found of the Appendices of this manual.

All samples introduced into the LIAB must have a barcode. If the sample does not have a barcode, the LIAB will not accept the sample.

The normal operating procedure for a sample run is as follows:

1. If there are any changes to the settings for the particular run they must be made before starting the LabView program. The functions of the different settings in the master program are described later in this manual. These settings will be in effect until the program is stopped and restarted. The program will then revert to the default settings. If there are a particular set of operations settings that are used on a routine basis, it is recommended that they be written down for future reference. The current version of LabView used to make the executable version of the Master Program does not allow changes to the default settings.

2. The program is started by clicking on the arrow in the upper left corner of the program screen. Once the master program is started, the sample is placed in the sample door and slowly rotated until the barcode is read. The unit will beep when it has read the barcode. The door will open and the sample will be accepted. If the unit is busy with other operations, it may take a few moments before the sample is taken into the unit.

3. Continue loading the samples into the unit until it is full or until the desired number of samples has been introduced. The front panel will indicate the sample status. This is described more fully below.

4. When the testing of a sample is complete, it will exit through the door in the bottom of the unit.

5. When all of the desired samples have been tested, the program should be stopped and the data can be retrieved from the $\mathrm{C}$ : lautosamplerldata directory. The program can be stopped by pressing the stop button in the lower right hand corner of the Monitor Screen.

\section{Data Files}

The data files that result from an analysis can be found in the directory C:lautosamplerldata. The naming convention for the data files is as follows:

User provided name-Dmonth_day_year-Thour_min_sec.csv

Where the date and time are the date and time that the file was created. All data until the program is stopped is written to this file. The naming convention prevents data from being overwritten and allows the files to be tracked by when they were created. The data files are flat text files with tab separators. A file utility has been provided with the master program to convert these files into comma separated files so 
that they can be imported directly into Excel. The files can be introduced into most other software using the import function. The setup of the headers is described in the section on the File Configuration Screen described below. In addition to the data provided below, the file setup uses the data in the NDIR configuration file (C: lautosampler/configuration \default.ndi) to create the headers for the NDIR channels. The wavenumbers for these channels have been pre entered in the File Configuration Screen.

\section{File Configuration Screen}

Figure 1 shows the file configuration screen. This screen is used to set the directory for data files, set the initial part of the data file name, to add comments to a data file and set the data file headers. Each function is described in more detail below.

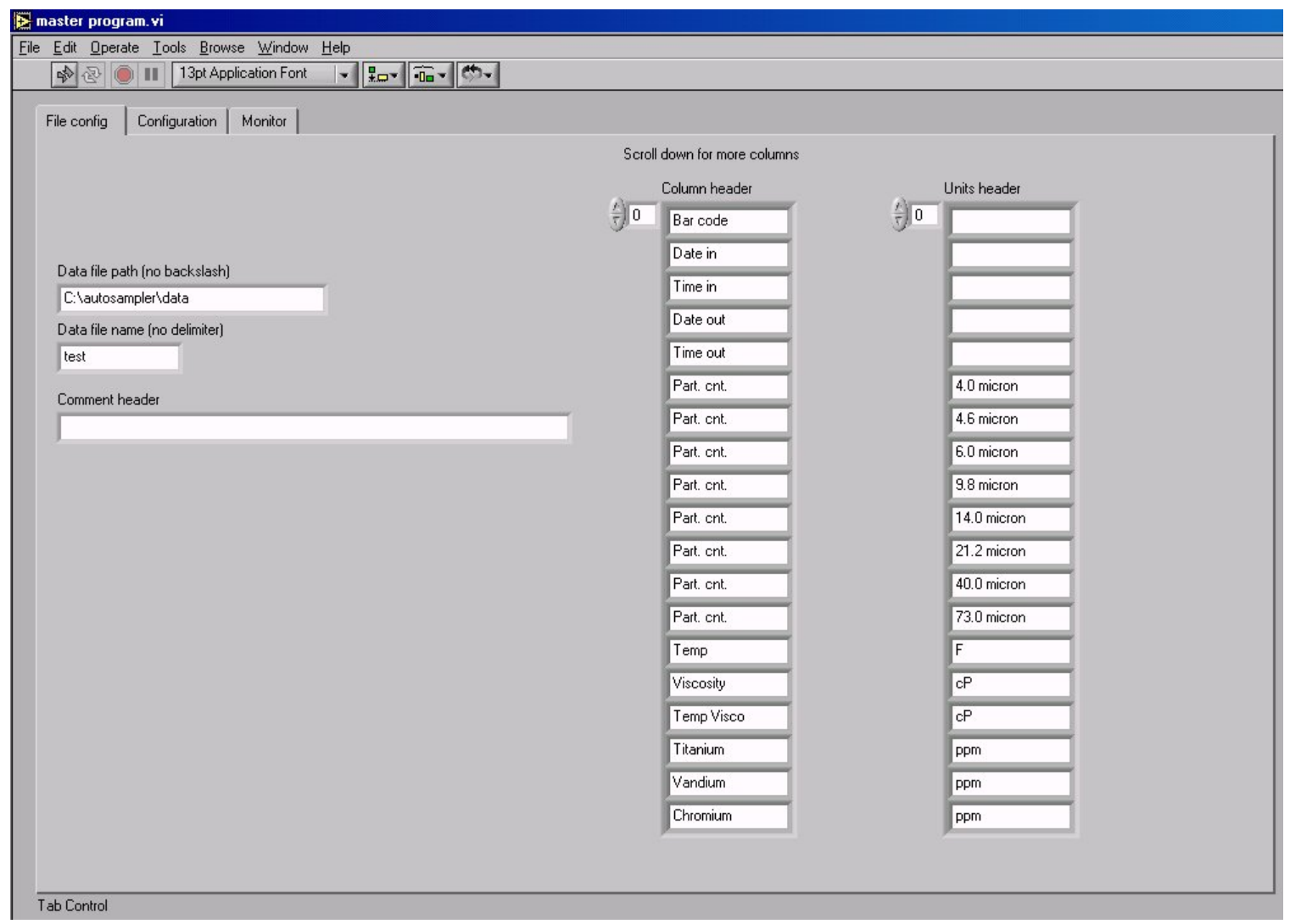

Figure 1. File configuration screen.

Data file path (no backslash). This sets the path where the data file will be written. The default is $\mathrm{C}$ : lautosamplerldata. If this default directory is changed it is important to insure that the directory exists or the program will give an error message.

Data file name (no delimiter). This sets the initial part of the data file name. The naming convention for data files is Data file name-Dmonth_day_year-Thour_minutes_sec.csv. An example of a data file name using the default value in the Data file name (no delimiter) field might be test-D04_14_2004T03_45_28.csv. Where the date 04/14/2004 is the date when the file was created and 03:45:28 is the 
hour, minute and second when the file was created. This convention is used to prevent the accidental overwriting of data files. A new file is created every time the program is restarted.

Comment header. This field allows comments to be placed into the first row of the data file.

Column header and Units header. This sets the headers for the data file. Under normal circumstances, these values will not be changed.

Note. The NDIR channels are contained in a separate file in the C: autosampler/configuration directory. The file is default.ndi and is preset to the customer selected NDIR channels. The wave numbers for these channels are preset in the units header column.

\section{Configuration Screen}

Figure 2 shows the configuration screen. This is the screen that you will see when the program first starts so that the operator can set the operating conditions before initiating a sample run. The screen is used to set the operating conditions for the instruments and the auto sampling unit. The default values on this screen are set to allow the unit to test the most viscous fluids that will be encountered in the customer's facility. These high viscosity fluids will take more time to draw from the sample bottle and be distributed to the instruments. If a batch of fluids with a lower viscosity is to be run, these values can be changed to decrease run time. The parameters are described below.

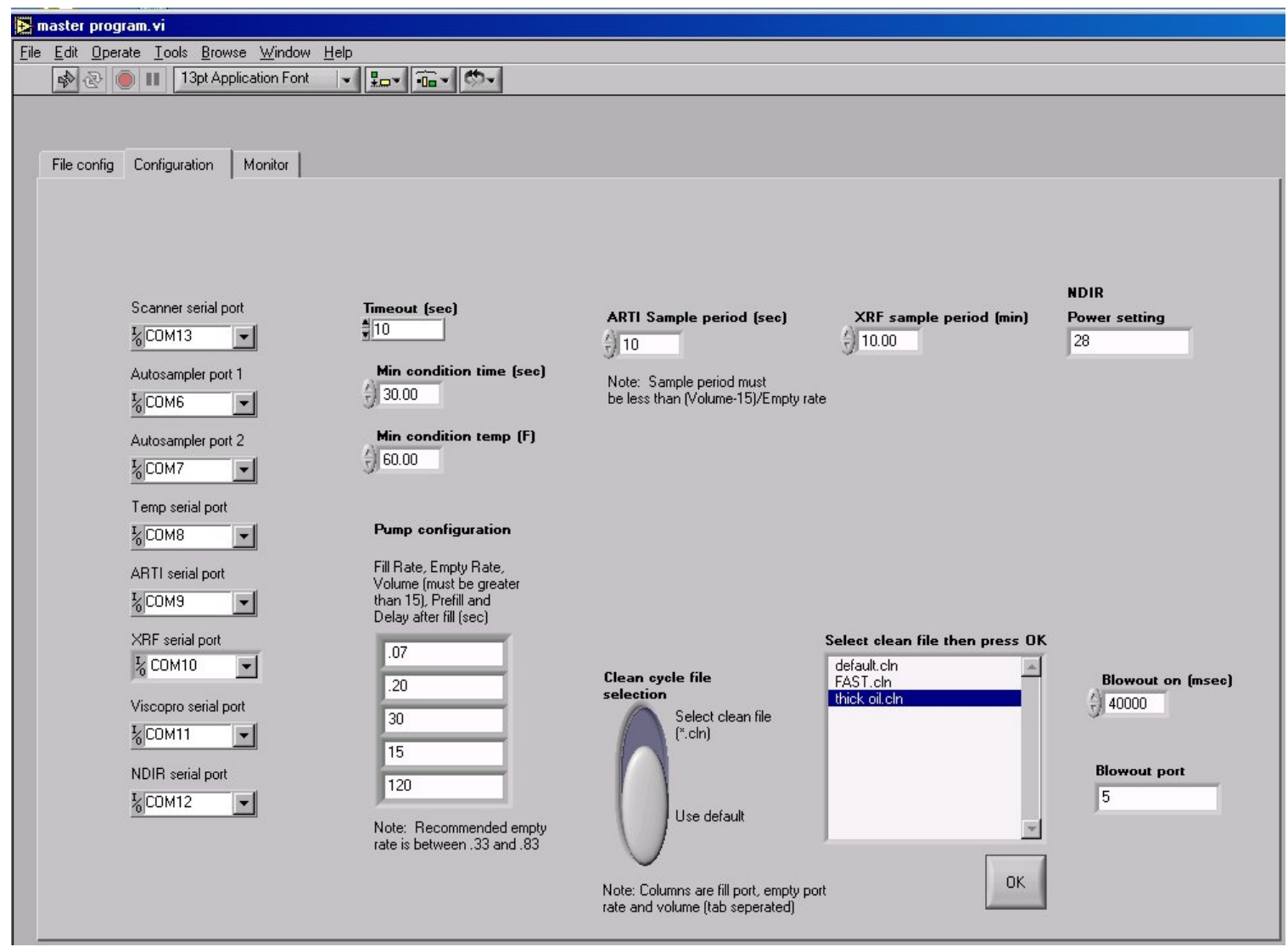

Figure 2. Configuration screen. 
Serial Port Settings. The left hand portion of this screen contains the serial port settings for each of the devices attached to the computer. These settings should not require changing. Settings for some of the instruments can be adjusted through these ports using HyperTerminal communication software and the serial commands in the attached manuals.

Timeout (sec). This sets the time that the program waits before reissuing commands in the event that there is a temporary break in communication. Generally this setting does not need to be changed.

Min conditioning time (sec) and Min conditioning temp (F). This sets the minimum time and temperature of the sample before it is allowed to leave the conditioning chamber. The best option in the event that the fluid is very cold is to adjust the conditioning time upward. The temperature in the conditioning chamber is maintained between 100-110 F.

Pump configuration. Using the proper pump configuration settings is extremely important to the proper operation of the instruments in the LIAB. There are 5 settings for configuring the pump. They are the fill rate of the pump $(\mathrm{ml} / \mathrm{sec})$, empty rate of the pump $(\mathrm{ml} / \mathrm{sec})$, sample volume $(\mathrm{ml})$, prefill $(\mathrm{ml})$, and delay after fill (sec). The fill rate and delay after fill insure that the sample to be tested is completely in the sample pump before dispensing the sample to the instruments. The default settings insure that the most viscous fluids can be properly sampled. If a series of fluids with lower viscosity are to be tested, the fill rate can be increased. The maximum allowable value for the fill rate is $15 \mathrm{ml} / \mathrm{sec}$. The empty rate is the rate at which the sample is dispensed to the instruments. The default setting allows the most viscous fluids to be dispensed to the instruments without stalling the pump. It is critical that the pump does not stall as this will affect the particle count and may result in fluid remaining in the chamber after the test. The optimal empty rate for the particle counter is between .33 and $.83 \mathrm{ml} / \mathrm{sec}$. The sample volume and prefill are set to insure that there is enough fluid for a complete test and that the particle counter is filled with fluid before starting a test. There should be no need to change these settings.

Arti Sampling Period (sec). This sets the sample period for the Arti Particle Counter. The sample period should be less than (fill volume-15)/empty rate. Normally there should be no need to change this setting.

XRF sample period (min). This sets the sample period for the XRF. Increasing the setting increases the accuracy of the metal contamination count. It may also need to be adjusted as the source in the XRF becomes depleted to obtain accurate measurements.

NDIR power setting. This sets the power for the NDIR. The setting should not be changed without consulting with the manufacturer of the NDIR as it is possible to damage the IR source if the setting is too high.

Clean cycle selection. This setting is used to select the cleaning cycle. If any cleaning cycle other than the default is going to be used, this must be set before running the program and the desired clean cycle selected from the list on the right. Clean cycles should have a cln extension to show up on the list. These files can be found in the directory $\mathrm{C}$ : $\backslash$ autosamplerlconfiguration. Each row in the clean cycle file sets the 
port that is used for filling the chamber, the port that the chamber is emptied into and the rate for the fill and empty. The default file has been designed to clean out every section of the instrument and may be used as an example creating a new file. Ports 3 and 4 are used for cleaning fluids. Port 5 is reserved for drawing air into the system to flush out any remaining fluid in the system. It is important that port 5 be kept clean as it can result in contamination of the samples if it is not. Figure 3 shows the fluid paths in the system and can be used to design a new clean cycle with the default clean cycle as a template. A normal clean cycle would draw air in through port 5 and send it through ports 0,1 and 2 to clean out residual fluid in the system. This would be followed by drawing cleaning fluid through either ports 3 or 4 and sent through ports 0,1 and 2 . Finally there should be at least one other air cycle to clean the cleaning fluid out of the system. The input file would look like the one shown below:

$\begin{array}{llll}5 & 0 & 15 & 100 \\ 5 & 1 & 15 & 100 \\ 5 & 2 & 15 & 100 \\ 3 & 0 & 15 & 100 \\ 3 & 1 & 15 & 100 \\ 3 & 2 & 15 & 100 \\ 5 & 0 & 15 & 100 \\ 5 & 1 & 15 & 100 \\ 5 & 2 & 15 & 100\end{array}$

The fill rate is $15 \mathrm{ml} / \mathrm{sec}$ and the volume is $100 \mathrm{ml}$ in each of the cycles.

Blowout on (msec) and Blowout port. These settings are used to complete the clean cycle by blowing pressurized gas through the instruments. The blowout on sets the time period for the blowout and the port setting makes sure that the air port 5 remains clean of residue. The on setting can be adjusted but the port setting should not be changed. 


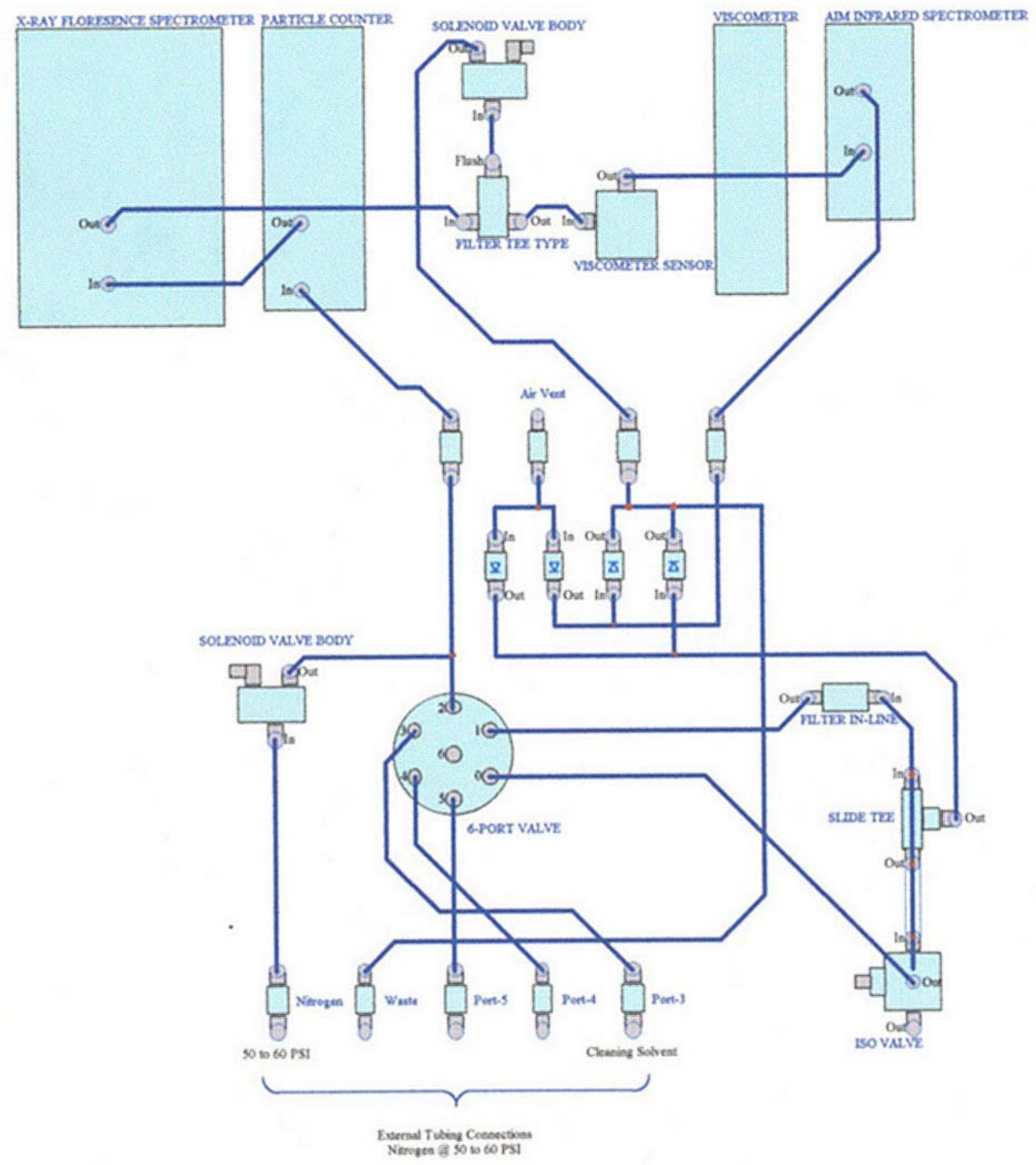

Figure 3. Tubing drawing of LIAB showing fill and empty ports. 


\section{Monitor Screen}

The monitor screen gives the status of the samples in the unit and the status of the instruments. In addition, it contains settings to unload the turret before starting a sample run and the option of turning off the clean cycle. Figure 4 shows the Monitor Screen.

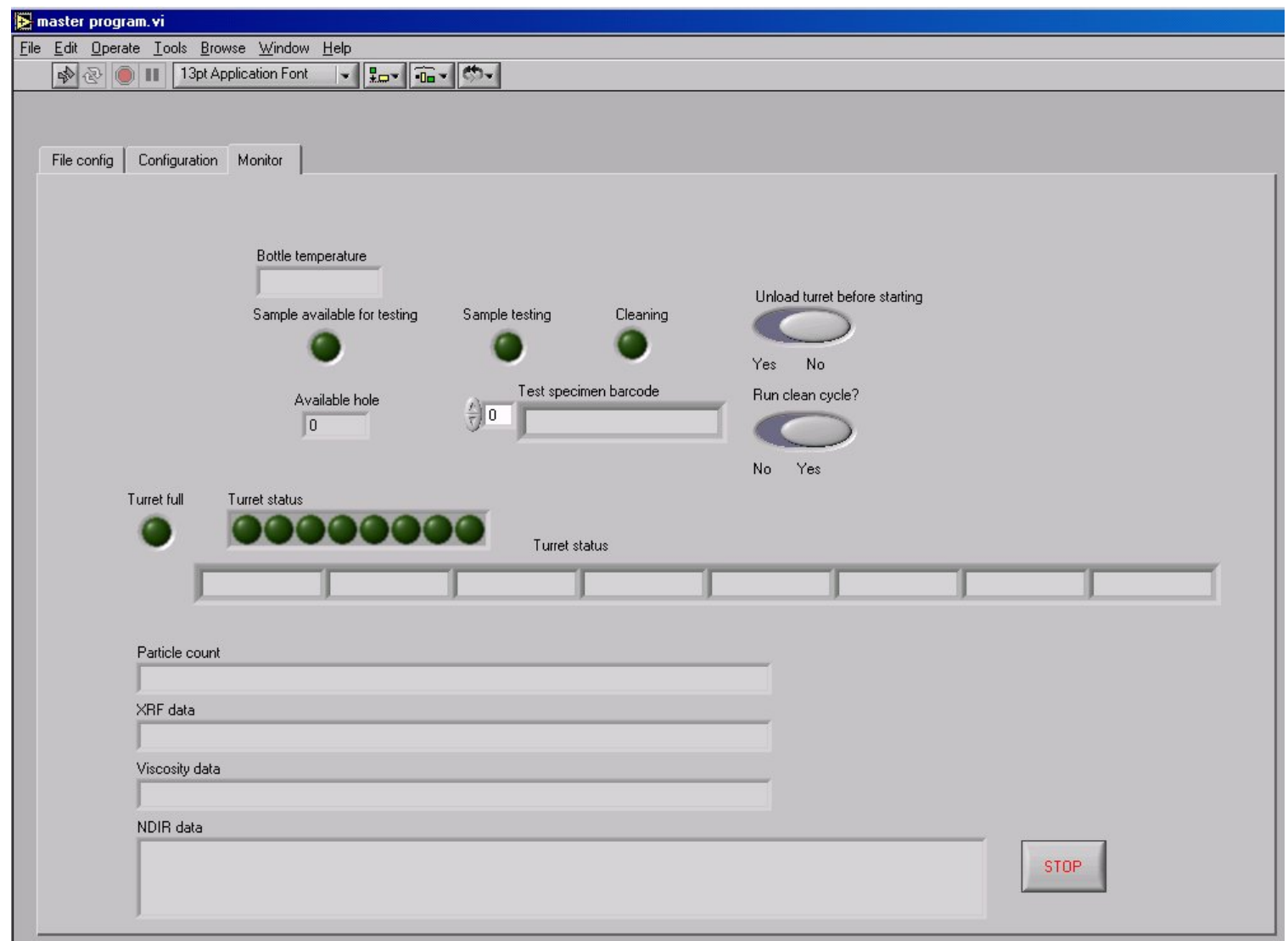

Figure 4. Monitor Screen.

Bottle temperature. This function is not implemented in this version of the software.

Sample available for testing, Sample testing and Cleaning. The first light indicates whether a sample has been conditioned long enough to be available for testing. The second light indicates whether a sample is being tested and the final light indicates if the sample is being cleaned. A sample cannot be testing while the clean indicator is on.

Available hole indicates the next available hole in the top turret that can accept a sample. Samples are tested on a first in first out basis.

Test specimen barcode tells the barcode of the sample that is being tested.

Turret full indicates that all 8 slots in the top turret have samples in them. 
Turret status indicates how many holes are full in the top turret of the sampler and gives the barcode of these samples.

Particle count, XRF data, Viscosity data and NDIR data show the last results of the last complete test. If the instrument does not produce data, the corresponding output will indicate that the instrument is off line.

Unload turret before start. If the unit crashes and the operator is uncertain whether there are still samples bottles in the unit, this can be set yes and the unit will unload all bottle holes in the top and bottom turrets before starting the normal sample cycle. This setting must be set before launching the program.

Run clean cycle? Normally this should be left to yes to insure that the machine is properly cleaned between samples. If multiple samples of the same specimen are to be run, this can be set to no to reduce the sample run time. This setting must be set before launching the program.

Stop. This button is used for the normal shutdown of the Master Program. It causes the orderly shutdown of the unit and is the preferred method of stopping the program. If this fails then the program can be aborted by pressing the stop sign in the upper left corner of the screen. This is not the recommended method of stopping the program as it does not result in an orderly shutdown of the unit. If the program is aborted, then it is necessary to cycle the power to the LIAB to insure that all of the subunits have been properly shutdown and restarted. Failure to do this can result in the LIAB in acting unpredictably since the microprocessors in the unit are not shut down when the abort is pressed. 


\section{Appendix A \\ Instrument Specifications/Calibration Sheets}




\title{
Arti Particle Counter
}

\section{Specifications \\ Light Extinction Sensor}

\author{
Sensitivity: $\quad 2 \mu \mathrm{m}$ (ISOMTD in oil) \\ Dynamic range: $\quad 2 \sim 100 \mu \mathrm{m}$ (ISOMTD in oil) \\ Flow Rate: $\quad 20-50 \mathrm{~mL} / \mathrm{min}$ \\ Max. Operating Pressure: $\quad 6000$ psi \\ Calibration: Calibrated by PSL in water and ISOMTD in oil \\ Resolution: $\quad \leq 10 \% @ 10 \mu \mathrm{m}$ with PSL calibration \\ Counting Efficiency: 30-70\%@2 $2 \mu \mathrm{m}, 90-110 \% @ 3 \mu \mathrm{m}$ (JIS) \\ Coincidence Loss: 10\% @ 30,000 particles/mL (PSL in water) \\ Wetted Materials: $\quad$ Sapphire and stainless steel \\ Light Source: $\quad$ Laser diode \\ Light Detector: $\quad$ PIN photo diode \\ Input Voltage: $\quad+5 \mathrm{VDC}$ \\ Dimensions: $\quad$ 2" diameter X 7" long \\ Environment: \\ Operating: 40 to $105^{\circ} \mathrm{F}\left(4\right.$ to $\left.41^{\circ} \mathrm{C}\right)$ (environment specs) \\ 55 to $105{ }^{\circ} \mathrm{F}\left(12\right.$ to $\left.41^{\circ} \mathrm{C}\right)$ (fluid specs) \\ 20 to $80 \%$ relative humidity, non-condensing
}

Storage: $\quad-40$ to $160{ }^{\circ} \mathrm{F}\left(-40\right.$ to $\left.70{ }^{\circ} \mathrm{C}\right)$

Up to $98 \%$ relative humidity, non-condensing 


\section{ARTI}

\section{Calibration Certificate}

This certifies the below mentioned Liquid Particle Counter System conforms to ISO11171:1999, Hydraulic Fluid Power Calibration of Automatic Particle Counters for Liquids. This system was calibrated with instruments and material traceable to international standards by factory authorized and trained technicians.

Note: The symbol $\mu \mathrm{m}(\mathrm{c})$ indicates ISOMTD particle size calibration according to ISO 11171:1999.

$\begin{array}{rc}\text { Date of Calibration : } & \text { October } 29,2003 \\ \text { Calibration Due Date: } & \text { October } 29,2004 \\ \text { Temperature: } & 22^{\circ} \mathrm{C}\left( \pm 1^{\circ} \mathrm{C}\right) \\ & \end{array}$

Instrument Model:

Serial Number: 30909407

Relative Humidity: $31 \%( \pm 7 R . H$.

Equipment used for calibration:

\begin{tabular}{|c|c|c|c|}
\hline Type & Model & Serial Number & Calibration Due Date \\
\hline PHA & RION KH-02A & 20280160 & 7 July 2004 \\
\hline $100 \mathrm{ml}$ Graduate & PYREX 3002-100 & $\mathrm{C} 28763$ & \\
\hline Stopwatch & VWR & MET0473 & 5 Feb. 2004 \\
\hline Reference PC & ARTI PODS & P1000-099 & 12 Feb. 2004 \\
\hline Reference Particles & NIST SRM-2806 & Lot \# 5-60-B & 31 Dec. 2003 \\
\hline
\end{tabular}

Instrument Calibration Data:

\begin{tabular}{|c|c|c|c|}
\hline Particle Size $(\mu \mathrm{m}(\mathbf{c}))$ & Threshold Setting (mV) & Particles Ref. Lot \# & Calibration Reference Material \\
\hline 4.0 & 55.5 & B101A & NIST 8631 Medium Test Dust \\
\hline 4.6 & 93.0 & B101A & NIST 8631 Medium Test Dust \\
\hline 6.0 & 255.0 & B101A & NIST 8631 Medium Test Dust \\
\hline 9.8 & 790.0 & B101A & NIST 8631 Medium Test Dust \\
\hline 14.0 & 1315.0 & B101A & NIST 8631 Medium Test Dust \\
\hline 21.2 & 2150.0 & B101A & NIST 8631 Medium Test Dust \\
\hline 40.0 & 2902.0 & 19989 & NIST Traceable Soda Lime Glass (DUKE) \\
\hline 73.0 & 3771.0 & 20283 & NIST Traceable Soda Lime Glass (DUKE) \\
\hline
\end{tabular}

Verification of Particle Counting Accuracy: (NIST RM 8632 ISOUFTD Lot \# A75)

\begin{tabular}{|c|c|c|}
\hline 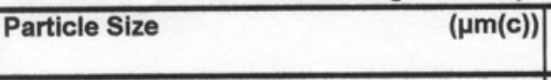 & $\begin{array}{c}\text { Expected Particle } \\
\text { Concentration }(\mathrm{p} / \mathrm{ml})\end{array}$ & $\begin{array}{c}\text { Observed Particle } \\
\text { Concentration }(\mathrm{p} / \mathrm{ml})\end{array}$ \\
\hline 3.0 & $8600-13000$ & 10669.0 \\
\hline 4.0 & $5400-8100$ & 7808.0 \\
\hline 5.0 & $3300-4500$ & 4484.0 \\
\hline 6.0 & $1500-2500$ & 2429.0 \\
\hline 10.0 & $58-220$ & 170.0 \\
\hline 14.0 & $4.1-19$ & 15.0 \\
\hline
\end{tabular}

Technician

D. W. Stantas 


\section{Non-dispersive Infrared Analysis}

The Non-dispersive infrared analysis (NDIR) is manufactured by Belhaven Applied Technologies. NDIR can measure infrared absorption for up to 32 channels in the 2.5 to 12 micron range (other ranges can be requested). Typical precision per band is given in Table 1 below.

\begin{tabular}{|l|l|l|l|l|}
\hline Wavelength (microns) & 0.8 & 2.9 & 5.7 & $6-9$ \\
\hline $\begin{array}{l}\text { Precision (average percent } \\
\text { error) }\end{array}$ & 0.4 & 0.013 & 0.23 & 0.62 \\
\hline
\end{tabular}

Table 1. Typical precision per band for non-dispersive infrared analyzer.

A comparison of NDIR results with standard laboratory measurements for water and TAN are given in Table 2 below. The NDIR is capable of measuring water content to $50 \mathrm{ppm}$ or less.

\begin{tabular}{|l|l|l|l|}
\hline System/Laboratory & NDIR Spectroscopy & Oil Analysis Lab* & Navy Lab** \\
\cline { 1 - 3 } Parameter & & & \\
\cline { 1 - 2 } Water Sample \#1 & $410 \mathrm{ppm}$ & $430 \mathrm{ppm}$ & $370 \mathrm{ppm}$ \\
Water Sample \#2 & $440 \mathrm{ppm}$ & $450 \mathrm{ppm}$ & $380 \mathrm{ppm}$ \\
\cline { 1 - 2 } TAN Sample \#1 & $0.70 \mathrm{mg} \mathrm{KOH} / \mathrm{g}$ & $0.70 \mathrm{mg} \mathrm{KOH} / \mathrm{g}$ & $0.64 \mathrm{mg} \mathrm{KOH} / \mathrm{g}$ \\
\cline { 1 - 2 } TAN Sample \#2 & $0.77 \mathrm{mg} \mathrm{KOH} / \mathrm{g}$ & $0.70 \mathrm{mg} \mathrm{KOH} / \mathrm{g}$ & $0.64 \mathrm{mg} \mathrm{KOH} / \mathrm{g}$ \\
\hline * Data provided by Oil Analysis Lab, Spokane, Wa \\
* Water by Karl Fischer, ASTM D1744, TAN by ASTM D664 \\
\hline **Water by Karl Fischer, ASTM D6304, TAN by ASTM D974 \\
\hline
\end{tabular}

Table 2. Comparison of NDIR results with laboratory measurements.

This unit is shipped with the following 5 filters as specified by Pall Corporation:

\begin{tabular}{|l|l|l|l|l|}
\hline Item Number & Wavelength & Wavenumber & Additive & $\begin{array}{l}\text { Additive } \\
\text { Wavenumber }\end{array}$ \\
\hline NB-2730-022 & $2730 \mathrm{~nm}$ & $3663 \mathrm{~cm}-1$ & phenolic antioxidant & $3648 \mathrm{~cm}-1$ \\
\hline NB-5730-080 & $5730 \mathrm{~nm}$ & $1745 \mathrm{~cm}-1$ & oxidation by-products & $1750 \mathrm{~cm}-1$ \\
\hline BP-8590-115 & $8590 \mathrm{~nm}$ & $1164 \mathrm{~cm}-1$ & sulfonate detergent & $1169 \mathrm{~cm}-1$ \\
\hline BP-9750-280 & $9750 \mathrm{~nm}$ & $1025 \mathrm{~cm}-1$ & $\begin{array}{l}\text { ZDDP }(\text { P-O-C) } \\
\text { additive }\end{array}$ & $978 \mathrm{~cm}-1$ \\
\hline
\end{tabular}




\section{ViscoPro 1500}

\section{General Specifications}

\section{Sensors}

Piston Range

Dynamic Range

Maximum Temperature

Maximum Recommended Pressure

Wetted Metals

\section{Electronics}

Temperature Units

Operating Temperature

Relative Humidity

Primary Power

\section{System}

Viscosity Accuracy

Viscosity Repeatability

Temperature Accuracy

Temperature Repeatability

Measurement Period
See Certificate of Calibration

20 to 1

$190^{\circ} \mathrm{C}\left(374^{\circ} \mathrm{F}\right)$

$1000 \mathrm{psi}$

316L and 430 Stainless Steel

Fahrenheit or Celsius

$10^{\circ} \mathrm{C}-65^{\circ} \mathrm{C}\left(50^{\circ} \mathrm{F}-150^{\circ} \mathrm{F}\right)$

$10-95 \%$, non-condensing.

18-36 VDC $\pm 1.0 \%$ Full Scale

$\pm 0.8 \%$ Reading ( $\pm 0.5 \%$ Typical $)$

$\pm 0.2^{\circ} \mathrm{C}$ within $20^{\circ} \mathrm{C}$ of Calibration Point(s)

$\pm 0.1^{\circ} \mathrm{C}$

13 seconds (typical) 


\subsection{Certificate of Calibration}

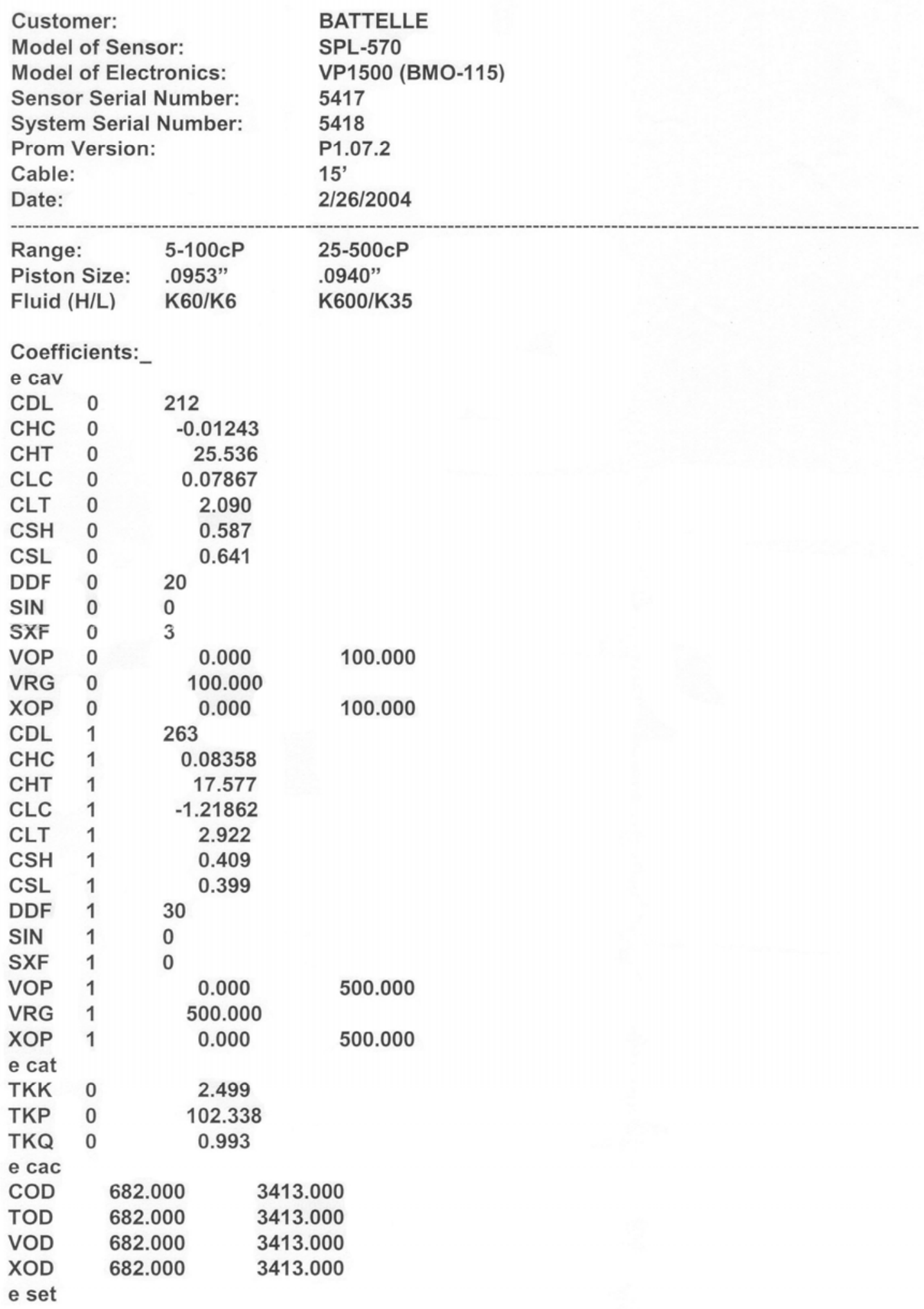




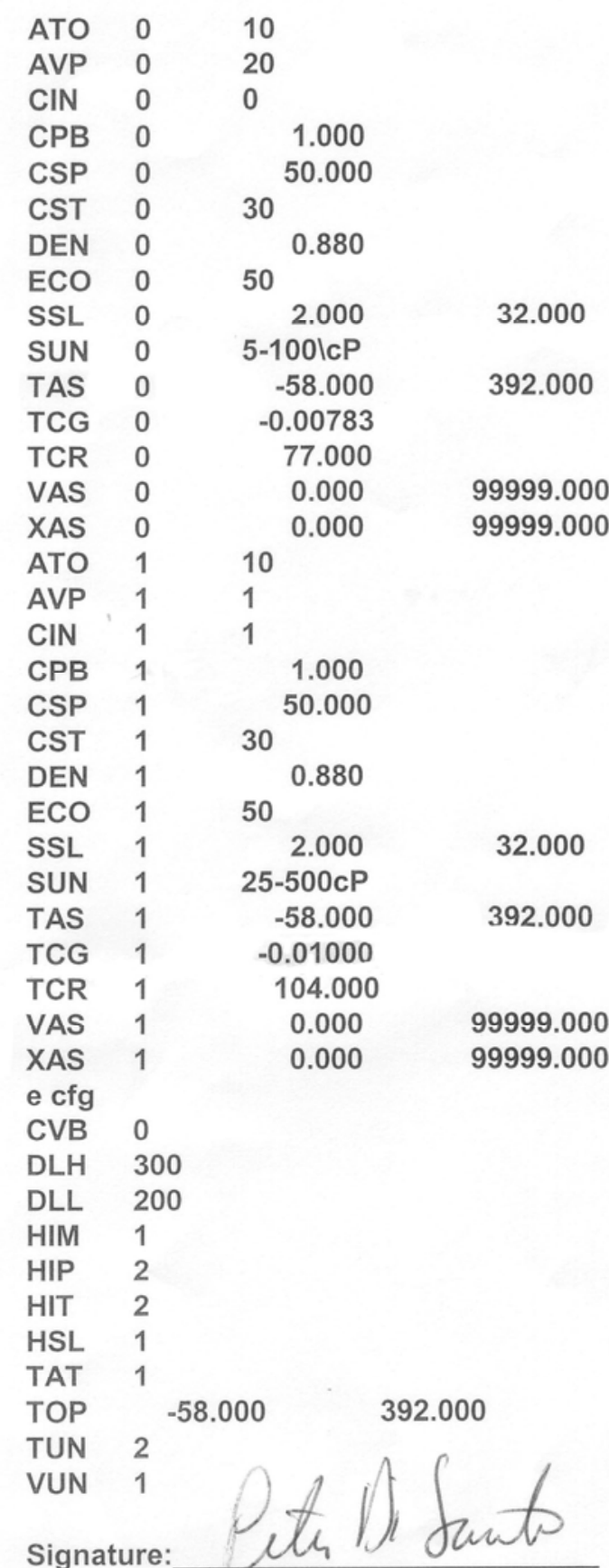

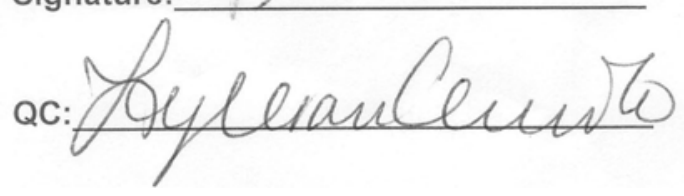

Date: $2-26-0 y$

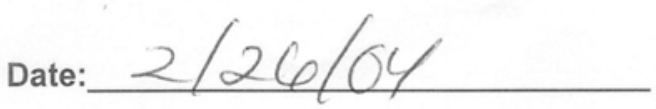




\section{X-ray Fluorescence Spectroscopy}

The standard source configuration for the XRF is ${ }^{109} \mathrm{Cadnium}$. There is no limit on the particle size for $\mathrm{x}-$ ray fluorescence spectroscopy measurements. Table 3 below lists the elemental detection limits at 10 percent precision for the standard configuration for the LIAB.

\begin{tabular}{|l|l|l|l|l|l|}
\hline Element & $\begin{array}{l}\text { Detection } \\
\text { Limit }(\mathbf{p p m})\end{array}$ & $\begin{array}{l}\text { AOAP } \\
\text { Calibration } \\
\mathbf{( p p m )}\end{array}$ & Element & $\begin{array}{l}\text { Detection } \\
\text { Limit } \mathbf{( p p m )}\end{array}$ & $\begin{array}{l}\text { AOAP } \\
\text { Calibration } \\
\mathbf{( p p m )}\end{array}$ \\
\hline Aluminum & $\mathrm{ns} *$ & 10 & Nickel & $5-10$ & 10 \\
\hline Boron & $\mathrm{ns}$ & 100 & Silicon & $\mathrm{ns}$ & 10 \\
\hline Chromium & $1-3$ & 10 & Silver** & $1-3$ & 10 \\
\hline Copper & $5-10$ & 10 & Sodium & $\mathrm{ns}$ & 10 \\
\hline Iron & $1-3$ & 10 & Tin & 10 & 10 \\
\hline Lead & $1-3$ & Titanium & $3-5$ & 10 \\
\hline Magnesium & $\mathrm{ns}$ & Zinc & $1-3$ & 100 \\
\hline Molybdenum & $3-5$ & 10 & & \\
\hline$*$ ns $=$ Not in standard configuration of XRF. & & \\
\hline ** Requires ${ }^{241}$ Americium source. \\
\hline
\end{tabular}




\section{Distribution}

No. of

Copies

\section{Onsite}

4 Pacific Northwest National Laboratory

L. O. Casazza (1) P7-07

A. F. Pardini (1) K5-26

M. L. Watkins (1) K5-26

W. C. Weimer (1) K9-09

Distr-1 